\title{
Joint state and parameter estimation for distributed mechanical systems
}

\author{
Philippe Moireau $\dagger$, Dominique Chapelle $\dagger^{*}$ Patrick Le Tallec $\ddagger$ \\ $\dagger$ INRIA, B.P. 105, 78153 Le Chesnay cedex, France \\ ¥Ecole Polytechnique, 91128 Palaiseau cedex, France
}

Comput. Methods Appl. Mech. Engrg. 197: 659-677, 2008, doi:10.1016/j.cma.2007.08.021

\begin{abstract}
We present a novel strategy to perform estimation for a dynamical mechanical system in standard operating conditions, namely, without ad hoc experimental testing. We adopt a sequential approach, and the joint state-parameter estimation procedure is based on a state estimator inspired from collocated feedback control. This type of state estimator is chosen due to its particular effectiveness and robustness, but the methodology proposed to adequately extend state estimation to joint stateparameter estimation is general, and - indeed - applicable with any other choice of state feedback observer. The convergence of the resulting joint estimator is mathematically established. In addition, we demonstrate its effectiveness with a biomechanical test problem defined to feature the same essential characteristics as a heart model, in which we identify localized contractility and stiffness parameters using measurements of a type that is available in medical imaging.
\end{abstract}

\section{Introduction}

The challenges represented by estimation in distributed mechanical systems have been recently renewed and extended - in particular - by the rapidly developing applications of biomechanics in medicine $[36,4,39]$. Such problems often involve three dimensional continuum mechanics coupled to biological phenomena and they can be represented by a set of coupled partial differential equations $[15,38,17]$. But the physical parameters considered in a biomechanical model are generally very difficult to determine a priori by experimentations, as living materials display very different behaviors when taken in vivo on the one hand, and post-mortem or even in vitro on the other hand. Moreover, for diagnosis purposes in medicine, estimation can be envisaged as a methodology to assess the condition of a patient's living organ. Therefore, there is a clear interest in being able to perform estimation using available measurements of the system in "standard" operating behavior - as opposed to ad hoc experimentations - measurements that for example can be provided by medical imaging. Note that this also holds, e.g., in meteorology or more generally in geophysics. In such fields the process aiming at obtaining unknown parameters - primarily initial conditions in the dynamical systems - by using various observational data is usually referred to as data assimilation.

As is well known, data assimilation methodologies fall into two main categories: variational and sequential (or filtering) procedures. Variational procedures consist in minimizing - with respect to all unknown

${ }^{*}$ Corresponding author: dominique. chapelle@inria.fr 
parameters - a criterion based on the observation error, with the model equations taken as constraints $[26,23,9,22]$. This leads to heavy computations in order to obtain the gradient of the criterion, generally by using the (backward in time) adjoint state, which requires extensive storage for large systems. On the other hand, sequential procedures in their classical forms - namely, Kalman filtering [21, 19, 8, 3], and related extensions to nonlinear systems - are not adapted to distributed mechanical systems because they involve the computation and manipulation - with some inversion steps - of covariance matrices that have the size of the state variable, just for estimating the state.

Regarding state estimation, however, we know from classical control and observation theory that it has close connections with control and stabilization [30]. Furthermore, since we are specifically concerned with mechanical systems, we can seek state estimators based on mechanical stabilization strategies that take into account the physical nature of the system at hand. In particular, collocated feedback is known to provide very effective stabilization strategies, both from theoretical (see [2] and references therein) and engineering perspectives [33], including with very "low-cost" (in terms of computations involved) feedback operators that can be directly used as filters in sequential state estimation.

The objective of this paper is thus to construct a joint state-parameter estimation procedure based on a simple collocated feedback strategy for state estimation, adequately extended by Kalman filtering techniques to allow the simultaneous estimation of a limited set of unknown parameters.

The outline of the paper is as follows. Section 2 describes the underlying physical models and the collocated feedback procedure which can be applied to estimate the state vector within the model. The performances of this estimation strategy is analyzed and numerically assessed in Section 3, with a detailed estimate of the damping properties of the proposed feedback. Section 4 introduces and analyses - both theoretically and numerically - a specific Kalman filtering technique coupled to the above feedback procedure for the joint state-parameter estimation in a linear framework. This methodology is then extended in Section 5 to a nonlinear estimation problem where the stiffness matrix depends linearly on unknown parameters.

\section{Problem statement}

\subsection{General framework}

We consider a mechanical system in the realm of solid or structural continuum mechanics, where the acceleration field inside the body is given by the imbalance between internal stresses and external forces. When $x$ denotes the state vector including displacements $y$ and velocities $\dot{y}$, such systems are described in a linear framework by a dynamical system - underlied by partial differential equations - written in the following generic form

$$
\left\{\begin{array}{l}
\frac{d x}{d t}=\mathcal{A} x+\mathcal{R} \\
x(0)=x_{0}+\zeta_{x}
\end{array}\right.
$$

where $\mathcal{A}$ is a linear differential operator generating a continuous semi-group, and $\mathcal{R}$ a source term. More specifically, this equation expresses the conservation of linear momentum, completed by the identity relating the velocity and the time derivative of displacement, namely, in a weak form,

$$
\begin{aligned}
& \int_{\Omega} \rho \frac{d \underline{y}}{d t} \cdot \delta \underline{y} d \Omega=\int_{\Omega} \rho \underline{\dot{y}} \cdot \delta \underline{y} d \Omega, \quad \forall \delta \underline{y} \\
& \left.\int_{\Omega} \rho \frac{d \underline{y}}{d t} \cdot \delta \underline{y} d \Omega=-\int_{\Omega} \underline{\Sigma} \underline{\underline{y}} \underline{\underline{y}}, \underline{\dot{y}}\right): \delta \underline{\underline{e}} d \Omega+\int_{\Omega} \underline{f} \cdot \delta \underline{y} d \Omega, \quad \forall \delta \underline{y}
\end{aligned}
$$


Here $\Omega$ represents the geometrical domain of the system, $\rho$ the mass per unit volume, $\underline{\underline{\Sigma}}$ the second PiolaKirchhoff stress tensor, $\delta \underline{y}$ an arbitrary test function in the displacement space with $\delta \underline{\underline{e}}$ the corresponding infinitesimal variation for the Green-Lagrange strain tensor, and $f$ the applied loading (taken here as a 3D distributed field to fix the ideas). Hence, in System (2.1) $x$ denotes the state variable $(\underline{y} \quad \dot{y})^{T}$. Assuming small displacements, we can identify $\delta \underline{\underline{e}}$ with the symmetric part of the gradient $\nabla \delta \underline{y}$, and take $\underline{\underline{\Sigma}}-$ which can then be identified with the Cauchy stress tensor - as a linear function of $x$. We are thus led to the linear operator $\mathcal{A}$. The differential system considered is of infinite dimension, its unknowns being the displacement and velocity fields at each point of the continuous body.

In the above system, $\zeta_{x}$ represents the unknown part in the initial condition $x(0)$. Likewise, we assume that $\mathcal{A}$ and $\mathcal{R}$ depend on a set of parameters in the form

$$
\theta=\theta_{0}+\zeta_{\theta}
$$

in which $\zeta_{\theta}$ is undetermined. Our objective is to obtain a joint estimation of the unknown quantities $\zeta_{x}$ and $\zeta_{\theta}$, based on measurements available for the system. These measurements are assumed to be given by

$$
Z=\mathcal{H} x+\chi
$$

where $\mathcal{H}$ is a linear operator referred to as the observation operator, and $\chi$ denotes a white noise introduced by the measurement procedure (detection, sampling...) that we describe in more details below. We also introduce

$$
\bar{Z}=\mathcal{H} x
$$

to represent an "exact measurement" which of course is never available in practice. More specifically, in the whole paper the measurements considered will be the velocities taken in a subpart $\Omega_{m}$ of the domain $\Omega$, and sampled in space by using weight functions $\left(s_{i}\right)_{i=1}^{q}$ defined on $q$ non-overlapping "measurement cells" within $\Omega_{m}$. Namely, $\mathcal{H} x=\left(0 \mathcal{H}^{v}\right)(\underline{y} \underline{\dot{y}})^{T}$ consists of the $q$ three-dimensional vectors given by

$$
\int_{\Omega_{m}} s_{i} \underline{\dot{y}} d \Omega
$$

and we assume that the sampling functions are normalized, i.e. $\left\|s_{i}\right\|_{L^{2}\left(\Omega_{m}\right)}=1$. We also assume that each of these measured vectors are independently perturbed with a white noise of associated covariance matrix $\alpha_{i}^{2} I_{3}$, which all together make up the above white noise $\chi$. Therefore, the covariance matrix of $\chi$ can also be decomposed in the following manner:

$$
Q_{\chi}=\sum_{i=1}^{q}\left(\alpha_{i}\right)^{2}\left[V_{i}^{1}\left(V_{i}^{1}\right)^{T}+V_{i}^{2}\left(V_{i}^{2}\right)^{T}+V_{i}^{3}\left(V_{i}^{3}\right)^{T}\right],
$$

where $V_{i}^{j}$ denotes the column vector with a 1 in the entry corresponding to the $j$-th coordinate (for $j=1,2,3$ ) of the $i$-th cell and 0 elsewhere.

Our estimation strategy will be based on observer theory and feedback control.

\subsection{Modelling and discretization}

Our observer approach relies on a discretized version of the above reference model. Namely, we approximate $x$ by $x_{h}$ where $x_{h}$ can be represented by a finite dimensional state vector $X$ of dimension $n$. The 
corresponding finite dimensional system can be written in the following formalism:

$$
\left\{\begin{array}{l}
\dot{X}=A X+R \\
X(0)=X_{0}+\zeta_{X}
\end{array}\right.
$$

where the initial condition expresses that $x_{h}(0)$ is the interpolation (or projection) of $x(0)$ in the finite dimensional subspace of the state space. We also introduce the operator $\mathfrak{J}_{h}$ as the one-to-one mapping from $\mathbb{R}^{n}$ to this subspace, namely, so that $x_{h}=\mathfrak{J}_{h} X$. Like the continuous formulation, this dynamical system represents the state space form of a variational - here discrete - formulation, typically derived from (2.2)-(2.3) by using a finite element discretization. Denoting by $Y$ the vector of degrees of freedom corresponding to the discrete displacement field, in the linear case the variational formulation yields a matrix equation of the type

$$
M \ddot{Y}+C \dot{Y}+K Y=F,
$$

where $M, C$ and $K$ respectively denote the mass, damping and stiffness matrices, and $F$ the load vector (see [7, 18]). With $X=\left(\begin{array}{ll}Y & \dot{Y}\end{array}\right)^{T}$, we thus have the following expressions for $A$ and $R$ in (2.7):

$$
A=\left(\begin{array}{cc}
0 & I \\
-M^{-1} K & -M^{-1} C
\end{array}\right), \quad R=\left(\begin{array}{c}
0 \\
M^{-1} F
\end{array}\right) .
$$

As a natural norm in the state space, we will use the energy norm, where the energy considered is the sum of kinetic energy and strain energy, namely,

$$
\|X\|_{\mathcal{E}}^{2}=\frac{1}{2} \dot{Y}^{T} M \dot{Y}+\frac{1}{2} Y^{T} K Y,
$$

and we define

$$
N=\frac{1}{2}\left(\begin{array}{cc}
K & 0 \\
0 & M
\end{array}\right)
$$

so that $\|X\|_{\mathcal{E}}^{2}=X^{T} N X$. Of course, the energy norm can also be considered for the continuous state, although not with the above matrix expressions.

As regards the observation, we have

$$
Z=H X+\epsilon_{h}+\chi
$$

with

$$
\begin{gathered}
H=\mathcal{H J}_{h}, \\
\epsilon_{h}=\mathcal{H}\left(x-x_{h}\right) .
\end{gathered}
$$

The quantity $\epsilon_{h}$ denotes a "small" approximation error produced by the discretization procedure in the observations.

Noting that $H$ only acts on velocities - like the continuous observation operator $\mathcal{H}$-we will also use the expression

$$
H=\left(\begin{array}{ll}
0 & H^{v}
\end{array}\right)
$$

From a practical point of view, $H^{v}$ consists of the collection of consistent force vectors $S_{i}^{j},(1 \leq i \leq$ $q, 1 \leq j \leq 3$ ), associated with the loading corresponding to a distributed force $s_{i}$ along the $j$-th cartesian coordinate, each of these vectors being stored in a separate line of $H^{v}$. 


\subsection{Model problem}

In order to illustrate and assess our estimation procedures, we will consider an example problem inspired from biomechanics and representing a simplified cardiac ventricle. The geometry of our example problem is depicted in Figure 1, and the characteristic dimensions of this object are - indeed - comparable to those of a human left ventricle. We thus resort to cardiac terminology to refer to the two extremities of the object, namely "apex and base" (see Figure). The system is clamped over the planar surface at the base, and activated by a planar wave of prestress - representing electrical activation - traveling from apex to base at wave speed $c=0.5 \mathrm{~m} \cdot \mathrm{s}^{-1}$, which means that it takes $0.2 \mathrm{~s}$ for the wave to reach the base. The wave shape itself is shown in Figure 2. The resulting prestress state is assumed to be isotropic and gives an external virtual work defined by

$$
\delta \mathcal{W}^{P S}=\sum_{1 \leq i \leq 17} \int_{\Omega_{i}^{A H A}} \theta_{i} \sigma_{0} w\left(x_{3}-c t\right) \operatorname{Tr}(\delta \nabla \underline{y}) d \Omega=\delta Y^{T} \cdot R,
$$

where the subdivision of the solid domain into 17 sub-regions is similar to the subdivision of the left ventricle advocated by the American Heart Association, see [1]. In the case of our simplified geometry this subdivision is depicted in Fig. 1 . In the above expression $\sigma_{0}$ denotes a constant contractility parameter, and $\theta_{i}$ a multiplicative coefficient that may take a different value in the range $[0,1]$ within each AHA region to represent pathological contraction. Namely, setting $\theta_{i}<1$ in a given region corresponds to a simplified model of infarcted tissue in that area, hence the parameters $\left(\theta_{i}\right)_{1 \leq i \leq 17}$ represent the quantities to be estimated for diagnosis purposes. In our reference simulations we take all these parameters to be 1 (healthy value) except for

$$
\theta_{14}=0.5
$$

see Figure 3.
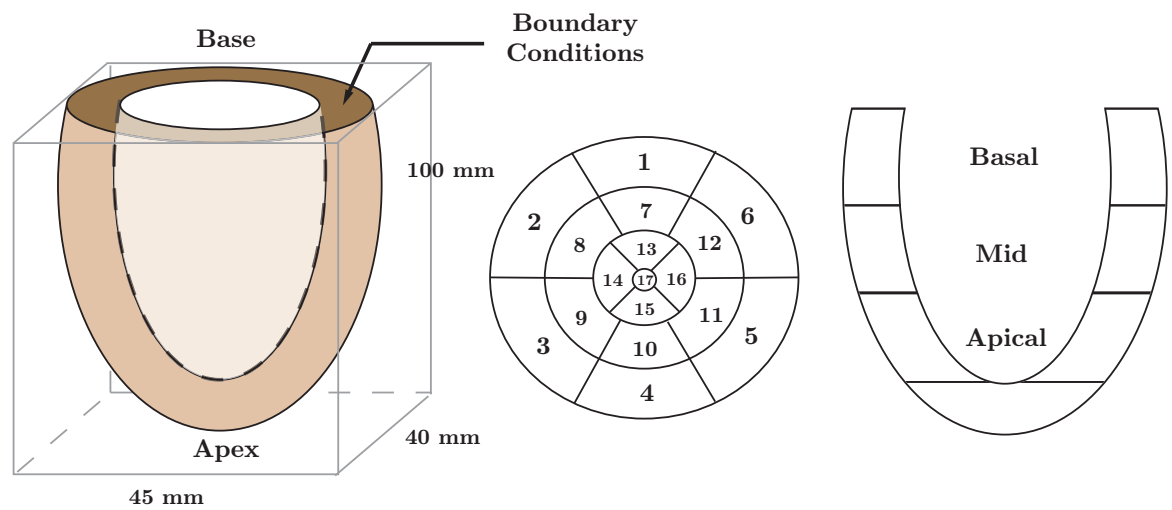

Figure 1: Model geometry (left) and AHA regions (center and right)

Our simulations will correspond to an isotropic viscoelastic material in linear analysis, with material parameters given by

$$
E_{i}=12.610^{3} \mathrm{~Pa}, \quad v_{\mathrm{i}}=0.3, \quad \eta_{\mathrm{i}}=0.227 \mathrm{~s} \quad \forall \mathrm{i} \in\{1, \ldots, 17\},
$$

and respectively denoting Young's modulus, the Poisson ratio and a viscoelastic coefficient associated with the pseudo-potential

$$
W^{v}=\eta_{i}\left(\frac{\lambda_{i}}{2}(\operatorname{Tr} \underline{\underline{\dot{\varepsilon}}})^{2}+\mu_{i} \operatorname{Tr}\left(\underline{\dot{\varepsilon}}^{2}\right)\right), \quad \forall i \in\{1, \ldots, 17\},
$$




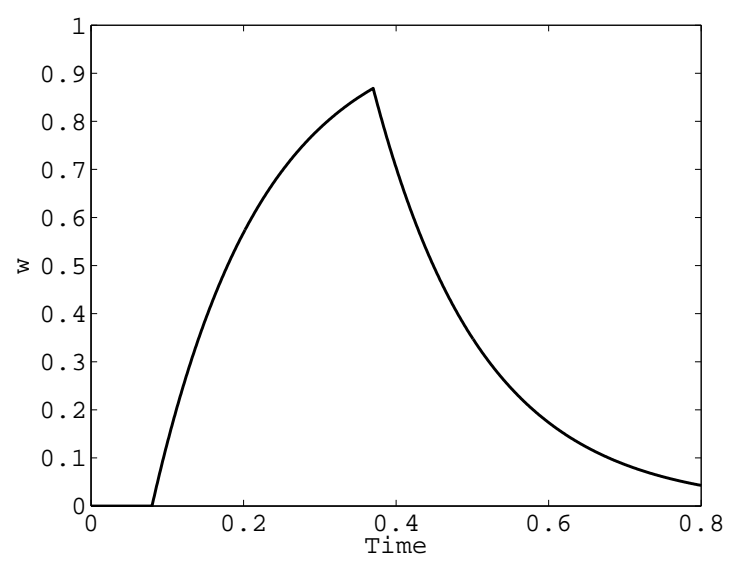

Figure 2: Activation profile $w$
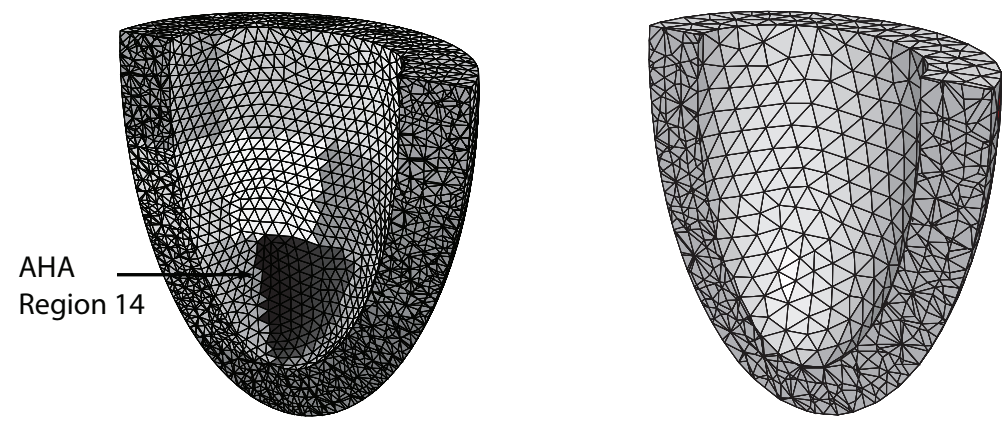

Figure 3: Reference mesh with 'infarcted' region (left) - 'Desired' mesh to be used in estimation (right)

where $\lambda_{i}$ et $\mu_{i}$ are the Lamé constants derived from $E_{i}$ and $v_{i}$, and

$$
\underline{\varepsilon}=\frac{1}{2}\left(\nabla \underline{y}^{T}+\nabla \underline{y}\right)
$$

denotes the linearized strain tensor approximating - at the first order - the Green-Lagrange deformation tensor in the small displacements framework. Note that this viscoelastic contribution corresponds to stiffness-based Rayleigh proportional damping. This leads to the following constitutive law to be taken into account in the variational formulation

$$
\underline{\underline{\Sigma}}=\lambda_{i} \operatorname{Tr}\left(\underline{\underline{\varepsilon}}+\eta_{i} \underline{\underline{\dot{\varepsilon}}}\right) \underline{\underline{1}}+2 \mu_{i}\left(\underline{\underline{\varepsilon}}+\eta_{i} \underline{\underline{\dot{\varepsilon}}}\right)
$$

Also, volumic mass is set as $\rho=10^{3} \mathrm{~kg} \cdot \mathrm{m}^{-3}$, a standard value for biological tissues.

The measurements used in the estimation procedures will be provided by a "reference model" given by a rather fine finite element discretization of the above object. The corresponding mesh is displayed in Figure 3 and features nearly 40000 degrees of freedom. The observer itself will be based on coarser discretizations, where the adequate mesh size will be a matter of discussion in the sequel. In all our simulations we used for time discretization the energy-conserving Newmark algorithm with time step 
$\Delta t=1 \mathrm{~ms}$. This time step is adequate for accurately representing the first 1000 eigenmodes of the system with at least 20 time steps per modal period, but is primarily determined in relation to the activation wave velocity. We show in Figure 4 the resulting energy and internal volume profiles for the reference simulations. Note that - unless otherwise stated - all physical units correspond to the SI system. We point out that internal volume is an important medical indicator and that our numerical values are "realistic" in this respect.

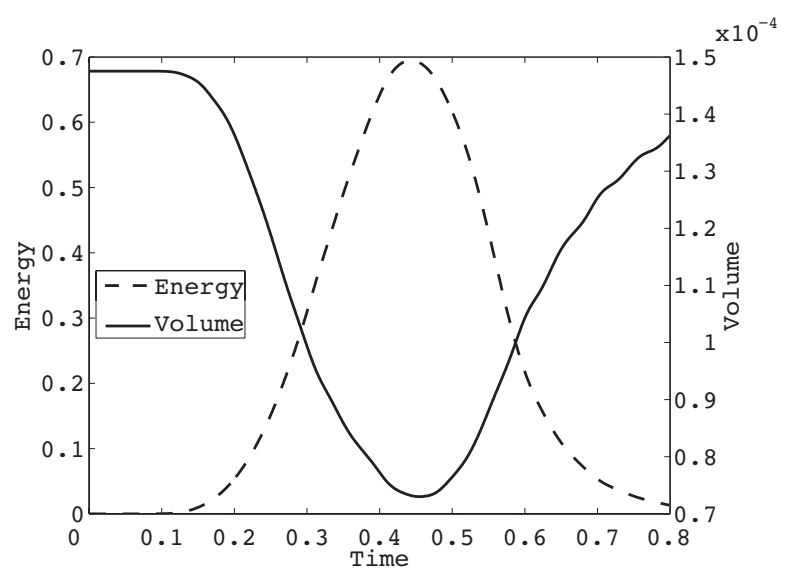

Figure 4: Energy and volume of the cavity for the reference solution

Finally, the measurement cells introduced above are defined by subdividing a (rectangular) box enclosing the geometry into $10 \times 10 \times 15$ smaller (rectangular) cells of equal sizes. This subdivision is visualized in Figure 5. The weight functions are then simply defined by scaled indicator functions of the cells. We point out that this resolution is comparable to that of standard tagged MRI images.

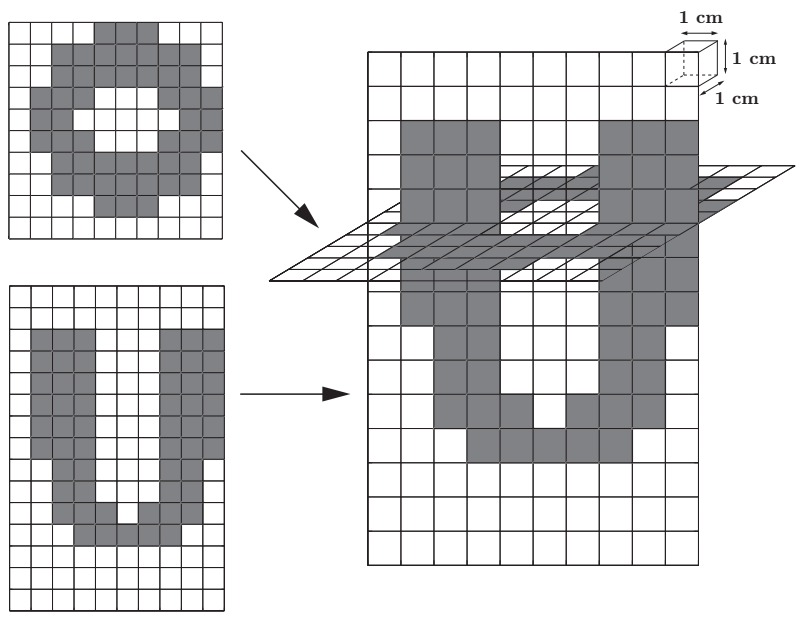

Figure 5: Measurement cells 


\subsection{State estimation using collocated damping}

We now introduce the finite dimensional state estimator of our original system (2.1)

$$
\left\{\begin{array}{l}
\dot{\bar{X}}=A \bar{X}+R+K_{X}(Z-H \bar{X}) \\
\bar{X}(0)=X_{0}
\end{array}\right.
$$

This state estimator uses the unbiased estimate of the initial condition and corrects the dynamics of the discrete system by a feedback proportional to the measured error. In essence, the filter $K_{X}$ that we want to use corresponds to a force proportional and opposed to the measured velocity, namely a "direct velocity feedback" (DVF) stabilization strategy, see [33, 10]. This is the simplest type of collocated feedback, namely a feedback law in which the control applied in a given location only uses the measurement corresponding to that location. In our case, the available measurements are weighted velocities within cells, hence inside each cell we want to apply a "filtering force" given by

$$
-\gamma s_{i} \int_{\Omega_{m}} s_{i} \dot{\dot{y}} d \Omega .
$$

Noting that the corresponding variational form of this feedback reads

$$
-\gamma \int_{\Omega_{m}} s_{i} \underline{\dot{y}} d \Omega \int_{\Omega_{m}} s_{i} \delta \underline{y} d \Omega,
$$

we infer that $K_{X}$ is simply given by

$$
K_{X}=\left(\begin{array}{ll}
0 & \gamma H^{v} M^{-1}
\end{array}\right)^{T} .
$$

Hence, the dynamics of the associated mechanical system is governed by

$$
M \ddot{\bar{Y}}+\left(C+\gamma\left(H^{v}\right)^{T} H^{v}\right) \dot{\bar{Y}}+K \bar{Y}=F+\gamma\left(H^{v}\right)^{T} Z,
$$

where the dissipative effect of the filter clearly appears through the positive factor $\gamma\left(H^{v}\right)^{T} H^{v}$. Note that this matrix is straightforward to compute in a mechanical finite element software, and that collocation induces a very narrow bandwidth, whereas Kalman filtering would yield full matrices.

\section{Analysis of the state estimator}

We point out that - in our mathematical error estimates - we use the symbol $C$ to denote a generic positive constant that may take different values for all occurrences.

\subsection{General framework}

Let us define the error $\check{X}$ between (2.7) et (2.17), namely,

$$
\check{X}=X-\bar{X},
$$

whose dynamics is given by

$$
\left\{\begin{array}{l}
\dot{\check{X}}=\left(A-K_{X} H\right) \check{X}-K_{X}\left(\epsilon_{h}+\chi\right) \\
\check{X}(0)=\zeta_{X}
\end{array}\right.
$$




\begin{tabular}{|c|c|}
\hline mesh & DOF $(\backsim)$ \\
\hline \hline Reference & $410^{4}$ \\
\hline Fine & $210^{4}$ \\
\hline Desired & $6.510^{3}$ \\
\hline Coarse & $2.910^{3}$ \\
\hline
\end{tabular}

Table 1: Number of dofs for the computational meshes

A straightforward computation shows that the covariance matrix of the vector $K_{X} \chi$ is of order $n$ - with $\mathrm{n}$ very large in finite element applications - and given by

$$
Q=\left(\begin{array}{cc}
0 & 0 \\
0 & \gamma^{2} \sum_{i=1}^{q} \sum_{j=1}^{3}\left(\alpha_{i}\right)^{2} M^{-1} S_{i}^{j}\left(S_{i}^{j}\right)^{T} M^{-1}
\end{array}\right) .
$$

Considering (3.1), in order to drive the above estimation error to zero, we are concerned with the properties - and more particularly the stability - of the dynamical system governed by $A-K_{X} H$, namely, the discretized form of a mechanical system with dissipative feedback. It is known that, under certain assumptions regarding the location and extent of $\Omega_{m}$, in the infinite dimensional case this closed-loop system is asymptotically exponentially stable in the energy norm, see in particular [27, 6]. However, such theoretical results do not provide quantitative estimations of exponential stability constants, which we are much concerned with. In addition, in our case the observer is based on a discrete system for which stability properties must be specifically investigated. We thus undertake this investigation by analysing the poles of the system considered, namely the eigenvalues of $\left(A-K_{X} H\right)$. As is well established in control theory (see [13] and references therein), this allows quantitative estimation of stability properties. Nevertheless, in our case a particular attention is necessary as to how these properties may be affected when changing the space discretization, since this constitutes a "modeling parameter" that may be varied in the observer formulation, in particular in order to improve the accuracy. Hence, we now present a numerical study of the poles for the above example problem with various values of $\gamma$ and several choices of discretizations.

We show in Figure 6 the root locus of the first 150 eigenmodes for four different values of the gain parameter $\gamma$, with a zoom near the imaginary axis in Fig. 7. These eigenpairs are computed with a mesh corresponding to about 6500 degrees of freedom - henceforth referred to as the "desired mesh" - but we show in Figure 8 that the dependence of the stabilization effect on the discretization is of no serious concern, see Table 1 for the sizes of the meshes considered. We point out that the "desired mesh" was deliberately selected with a limited number of degrees of freedom in order to provide fast computations in the estimation procedure, while representing a "reasonable" discretization of the geometry, see Figure 3. Of course, we will be concerned with the errors induced by this discretization, much coarser than that used in the reference simulations, and we will assess these discretization errors in our forthcoming analyses.

\subsection{Damping properties of the collocated system}

Although the above numerical results indicate a very effective stabilizing effect for our collocated feedback, these results only concern - of course - a limited number of poles, and - furthermore - provide no information on the associated eigenvectors, in particular as to how well they "span" the state space. Therefore, they only give an incomplete description of the dynamics of the error system (2.18). For a 


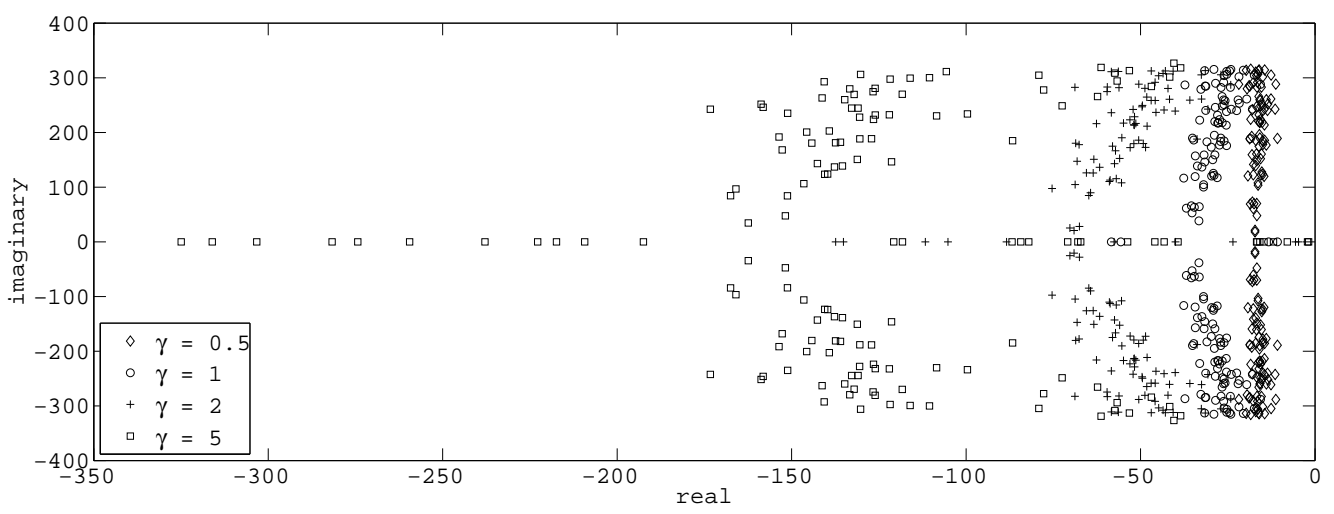

Figure 6: Root locus for different values of $\gamma$ (units in $\mathrm{s}^{-1}$ )

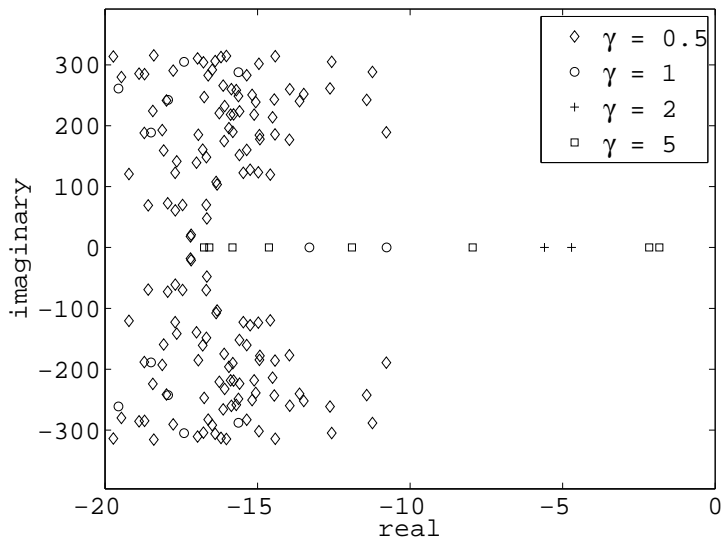

Figure 7: Zoom on the root locus near imaginary axis 

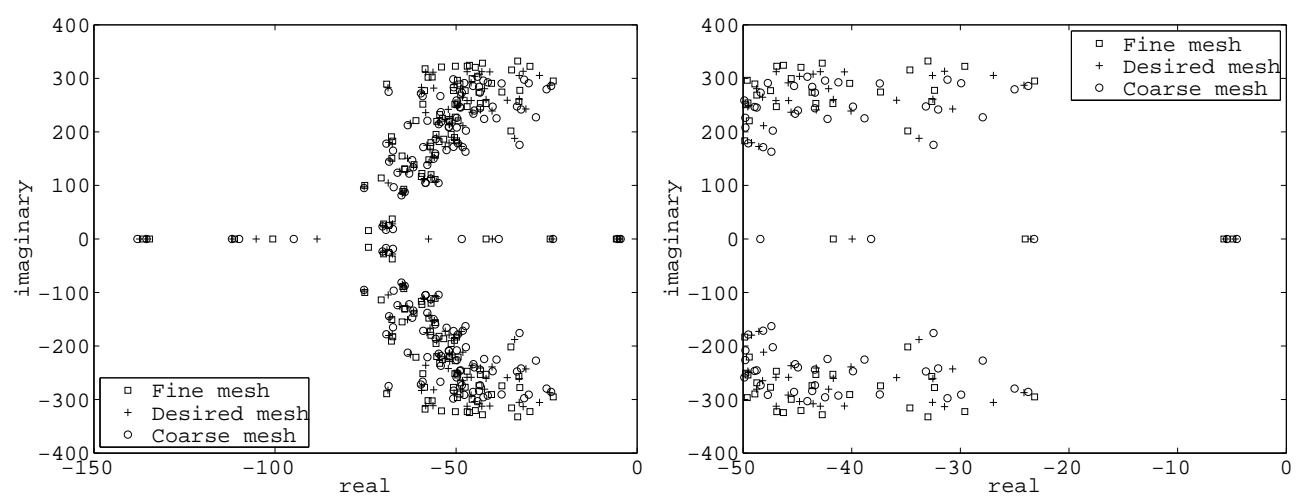

Figure 8: Stability of the root locus w.r.t. mesh discretization (near imaginary axis)

continuous system, a desirable related feature is that the eigenvectors make up a Riesz base [13, 37]. This can be mathematically established for the corresponding one-dimensional problem, see [12]. However, there is no such general result in $2 \mathrm{D}$ or $3 \mathrm{D}$ or in elasticity, in particular due to geometrical effects $[24,25]$. Nevertheless, for practical purposes it is not necessary to take into account the full complexity of the spectral problem - and in particular as regards the behavior of the numerical and physical high frequencies and modes - in order to evaluate the rate of exponential stability for the sequence of homogeneous dynamical systems

$$
\left\{\begin{array}{l}
\dot{\check{X}}_{h g}=\left(A-K_{X} H\right) \check{X}_{h g} \\
\check{X}_{h g}(0)=\zeta_{X}
\end{array}\right.
$$

Indeed, a "real" initial condition $x(0)=x_{0}+\zeta_{x}$ is in general sufficiently regular to be well approximated by only "a few" eigenmodes of the system without stabilizer. The discrete solution at any time can then be bounded using the following result, where we denote by $\left(\Phi_{i}, \lambda_{i}\right)$ and $\left(\Psi_{i}, \mu_{i}\right)$ the complex eigenpairs of $A$ and $A-K_{X} H$, respectively - assuming that these eigenpairs are arranged in ascending order of the associated eigenfrequencies.

\section{Proposition 3.1}

Assume that for System (3.3) the initial condition can be expanded in the modal basis $\left\{\Phi_{i}\right\}$ of the original system as

$$
\check{X}_{h g}(0)=\sum_{i=1}^{q} \alpha_{i} \Phi_{i}+r_{q} .
$$

Then, for any time $t$ we have the bound

$$
\left\|\check{X}_{h g}(t)\right\|_{\mathcal{E}} \leq C\left(q^{\prime}\right) e^{-\delta\left(q^{\prime}\right) t}\left(1+d\left(q, q^{\prime}\right)\right)\left(\left\|\check{X}_{h g}(0)\right\|_{\mathcal{E}}+\left\|r_{q}\right\|_{\mathcal{E}}\right)+\left\|r_{q}\right\|_{\mathcal{E}}+d\left(q, q^{\prime}\right)\left(\left\|\check{X}_{h g}(0)\right\|_{\mathcal{E}}+\left\|r_{q}\right\|_{\mathcal{E}}\right),
$$

where the constants appearing in this estimate are defined as follows,

$$
\begin{gathered}
\delta\left(q^{\prime}\right)=\inf _{i \leq q^{\prime}}\left(-\mathfrak{R}\left(\mu_{i}\right)\right), \\
d\left(q, q^{\prime}\right)=\sup _{V \in \operatorname{span}\left(\Phi_{i}\right)_{i=1}^{q}} \inf _{W \in \operatorname{span}\left(\Psi_{i}\right)_{i=1}^{q^{\prime}}} \frac{\|V-W\|_{\mathcal{E}}}{\|V\|_{\mathcal{E}}},
\end{gathered}
$$




$$
C\left(q^{\prime}\right)=\frac{\gamma_{2}\left(q^{\prime}\right)}{\gamma_{1}\left(q^{\prime}\right)} \quad \text { s.t. } \gamma_{1}\left(q^{\prime}\right) \sum_{i=1}^{q^{\prime}}\left|\alpha_{i}\right| \leq\left\|\sum_{i=1}^{q^{\prime}} \alpha_{i} \Psi_{i}\right\|_{\mathcal{E}} \leq \gamma_{2}\left(q^{\prime}\right) \sum_{i=1}^{q^{\prime}}\left|\alpha_{i}\right| .
$$

The estimate (3.4) is straightforward to establish, and it means that, once the initial condition is decomposed using the modes of the original system (up to some "small" remainder $r_{q}$ ), the state at all times can be bounded using the poles of the collocated system with $\mathfrak{R}\left(\mu_{i}\right)$ the real parts of the corresponding eigenfrequencies. The constant $d\left(q, q^{\prime}\right)$ represents the distance between the subspaces spanned by the eigenvectors $\left\{\Phi_{i}\right\}$ and $\left\{\Psi_{i}\right\}$ of the original and collocated systems, and $C\left(q^{\prime}\right)$ the constant of equivalence of norms between the energy norm and the norm associated with modal components. It can be checked numerically (by computing the corresponding Rayleigh quotients) that $d\left(q, q^{\prime}\right)$ is "small" as soon as $q^{\prime} \geq q$, and that $C\left(q^{\prime}\right)$ remains finite, both properties being obtained rather independently of the mesh considered. This can be seen as a numerical verification of the fact that the collocated eigenvectors adequately span the state space - the numerical counterpart of the above-mentioned Riesz basis property, valid at least for the low frequencies which are the main concern in our case.

Hence we are led to an estimate of the type

$$
\left\|\check{X}_{h g}(t)\right\|_{\mathcal{E}} \leq C_{1} e^{-\delta_{1} t}\left\|\check{X}_{h g}(0)\right\|_{\mathcal{E}}+\varepsilon,
$$

where $\delta_{1}$ is related to the poles positions of the collocated system, and $\varepsilon$ denotes a small quantity. Our aim is that $\delta_{1} . T>>1$, which expresses that the exponential stability is meaningful with respect to the time constant of the dynamics. In other words, we want to estimate quantitatively the time constant of the stability, and analysing the poles is a way to achieve such an estimation whereas abstract results on exponential stability usually just provide the existence of such an exponential estimate. In this respect, only limited semi-quantitative results are available - see e.g. [33] - such as the following expression for the sensitivity of the poles position with respect to $\gamma$

$$
\frac{d \mu_{i}}{d \gamma}(0)=\frac{d \mathfrak{R}\left(\mu_{i}\right)}{d \gamma}(0)=-\frac{W_{i} H^{T} H W_{i}}{2}
$$

where $W_{i}$ denotes the $i$-th mass-normalized displacement eigenvector of the original system, namely such that

$$
K W_{i}=\lambda_{i} M W_{i}, \quad W_{i}^{T} M W_{i}=1 .
$$

We now illustrate this discussion with some numerical simulations obtained with our example problem. Figure 9 displays the effect of an error in the initial condition corresponding to a static displacement obtained by imposing an internal pressure of $10^{3} \mathrm{~Pa}$, which is the order of magnitude of the pressure induced by atrium contraction during ventricular filling. The quantity shown is the energy (in base 10 $\log$ ) of the simulated state corresponding to System (3.3). We can distinguish two parts in the resulting evolutions, corresponding to two clearly distinct stabilization rates for each choice of $\gamma$. The first slope is related to the eigenmodes which dominate in the initial condition, while the second one corresponds to the least stable poles that are led to the real axis due to the damping added by the collocated stabilizer, recall Figs. 6-7. Note that the asymptotic behavior may also be conditioned by some high frequencies - whether they be physical or purely numerical as discussed in [5] - which is not observed here. We can see in Figure 9 that the choice $\gamma=2$ is optimal as regards the initial slope, and that the corresponding asymptotic slope is associated with errors that are of no practical concern.

Unless otherwise indicated, we will use the static displacement produced by the above internal pressure as the initial state error in our numerical estimation experiments in the sequel. 


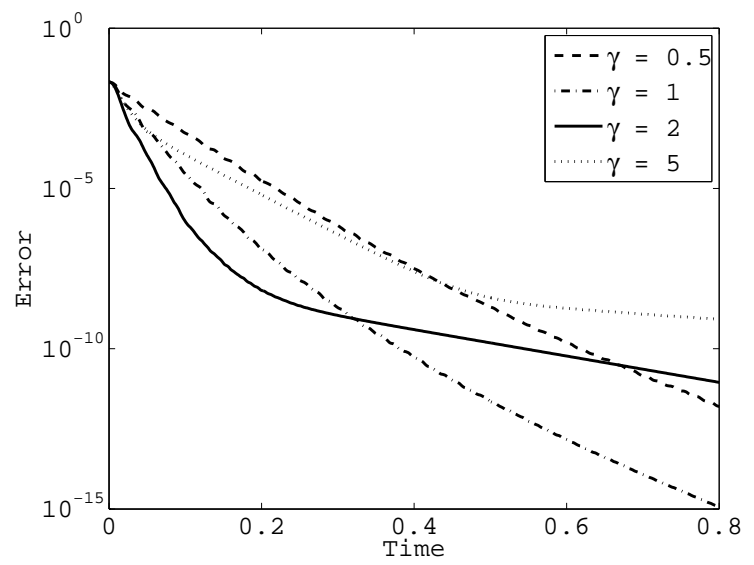

Figure 9: Energy for initial condition error (log scale)

\subsection{Influence of measurement and discretization errors}

Let us now concentrate on the influence of the right-hand side $K_{X}\left(\epsilon_{h}+\chi\right)$. In this matter, we distinguish between the deterministic term $K_{X} \epsilon_{h}$ and the probabilistic one $K_{X} \chi$. The main difference with our previous analysis is that we cannot assume any space regularity on these terms and they may - indeed - include some high frequency contents. As a consequence, we will consider both the case of uniformly exponential stability and the case where we only have stability in the error system (3.1). To that end we define - independently of the discretization in all cases - two positive constants $\delta$ and $C$ such that:

$$
\left\{\begin{array}{l}
\delta . T>>1 \text { if uniformly exponential stability } \\
\delta=0 \quad \text { if only stability }
\end{array}\right.
$$

This allows to bound the (discrete) semi-group $\mathcal{T}^{h}(t)$ generated by the operator $\left(A-K_{X} H\right)$ as

$$
\left\|\mathcal{T}^{h}(t)\right\| \leq C e^{-\delta t}
$$

which in turn will provide bounds on the errors.

We first consider the effect of the probabilistic term $K_{X} \chi$. To that purpose we can analyze the dynamical system

$$
\left\{\begin{array}{l}
\dot{\check{X}}_{\chi}=\left(A-K_{X} H\right) \check{X}_{\chi}-K_{X} \chi \\
\check{X}_{\chi}(0)=0
\end{array}\right.
$$

The random variable $\check{X}_{\chi}$ is then Gaussian, hence characterized by the behavior of its mean and covariance. We obviously have $\mathbb{E}\left(\check{X}_{\chi}\right)=0$ for the mean. In order to deal with the covariance we use the semi-group formulation and obtain

$$
\mathbb{E}\left(\left\|\check{X}_{\chi}\right\|_{\mathcal{E}}^{2}\right)=\int_{0}^{T} \operatorname{Tr}\left(Q\left(\mathcal{T}^{h}(t-\tau)\right)^{T} N \mathcal{T}^{h}(t-\tau)\right) d \tau
$$

where $Q$ is the covariance of the white noise $K_{X} \chi$ and $N$ is the energy norm matrix. Substituting the expression in (3.2) and using (3.7), we directly obtain

$$
\mathbb{E}\left(\left\|\check{X}_{\chi}\right\|_{\mathcal{E}}^{2}\right) \leq C_{2} T_{2} \gamma^{2} \sum_{i=1}^{q} \alpha_{i}^{2}
$$


with

$$
T_{2}=\left\{\begin{array}{l}
1 / \delta \ll T \quad \text { if uniformly exponential stability } \\
T \quad \text { if only stability }
\end{array}\right.
$$

The influence of observation noise is illustrated with our example problem. We took a white noise standard deviation corresponding to ten percent of a reference velocity value in our simulations for a sampling rate of $50 \mathrm{~ms}$. When rescaled according to white noise rules to the actual computational time step, this corresponds to a standard deviation of about 70 percent of the reference value. We show in Figure 10 the energy of the solution $\check{X}$ obtained with various values of the observer gain $\gamma$. As expected

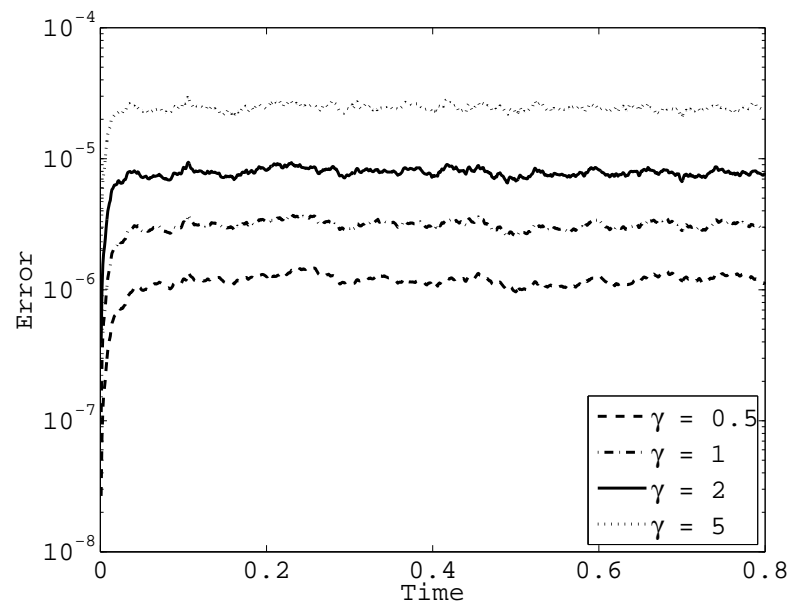

Figure 10: Error induced by measurement noise (energy in log scale)

from the above numerical analysis, the gain has an amplification effect on the noise which is of the type predicted by (3.9). Nevertheless, we can see that for all values of $\gamma$ of interest, the error associated with the noise is rather small - and indeed not dominant compared to the modeling error that we will now investigate.

We thus complete our stability analysis with the study of the the deterministic source term $K_{X} \epsilon_{h}$. Namely, we consider the system

$$
\left\{\begin{array}{l}
\dot{\check{X}}_{d}=\left(A-K_{X} H\right) \check{X}_{d}-K_{X} \epsilon_{h} \\
\check{X}_{0}=0
\end{array}\right.
$$

Using Duhamel's formula we obtain

$$
\check{X}_{d}(t)=\int_{0}^{t} \mathcal{T}^{h}(t-\tau) K_{X} \epsilon_{h}(\tau) d \tau
$$

and we infer the following estimation for the deterministic right-hand side

$$
\left\|\check{X}_{d}(t)\right\|_{\mathcal{E}} \leq C \gamma \sqrt{T_{2}}\left\|x-x_{h}\right\|_{L^{2}([0, T] ; \mathcal{E})} .
$$

We can summarize the convergence of our state estimator in the following estimate combining the contributions of initial error, discretisation error, measurement noise, and high frequency cut-off.

$$
\mathbb{E}\left(\|\check{X}\|_{\mathcal{E}}^{2}\right) \leq C\left[e^{-2 \delta_{1} t} \mathbb{E}\left(\left\|\zeta_{X}\right\|_{\mathcal{E}}^{2}\right)+T_{2} \gamma^{2}\left\|x-x_{h}\right\|_{L^{2}([0, T] ; \mathcal{E})}^{2}+T_{2} \gamma^{2} \sum_{i=1}^{q} \alpha_{i}^{2}+\varepsilon^{2}\right] .
$$


We give the corresponding numerical results in Figure 11, where we clearly see that the modeling error - here due to the discretization - is dominant in the error after the initial condition error has been damped according to the above discussion. As expected this modeling error only arises with the occurrence of the activation wave. We also remark that the modeling error effect is quite stable in the range of $\gamma$ chosen, and that we do not observe the amplification effect of $\gamma$ that could be expected from (3.13).

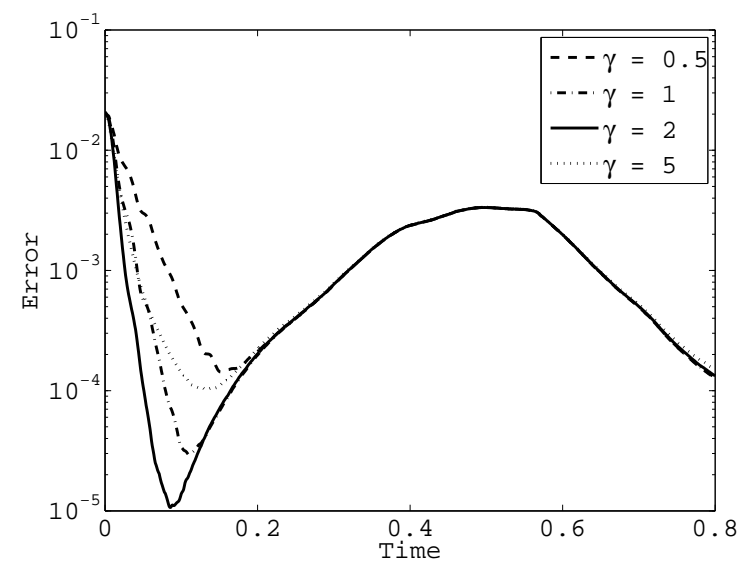

Figure 11: Global error with respect to $\gamma$ (energy in log scale)

Eventually, in order to assess the dependence of our procedure with respect to the discretization, we give in Figure 12 the results obtained with observers based on three different meshes - recall Table 1 with measurements generated from the same above-defined reference mesh. We conclude that the effect of the discretization is roughly as predicted from (3.13). Also, we compare in Figure 13 the estimation error for the desired mesh with the errors associated with

- the solution of the direct problem (2.7) generated with the desired mesh without initial condition error (this gives the curve labeled "discretization error" in Fig.13);

- the reference solution interpolated in the desired mesh at each time step (the "interpolation error").

This comparison shows that - after a short time corresponding to the stabilization of the collocated system - the observer provides an estimation with optimal accuracy as compared with the interpolation error. The estimated state is indeed superior to the solution directly simulated on the desired mesh for the period of maximum energy, despite the very small time step that reduces - in the direct solution - the error due to the approximation of high frequency dynamics. This - of course - is only possible because of the feedback provided by the measurements in the observer.

\section{Joint state-parameter estimation: the linear case}

\subsection{General framework}

In this section, we consider the estimation of parameters only included in the right-hand side of the dynamical equation, and with a linear dependence on the parameter vector $\theta$ of dimension $r$. Hence, we 


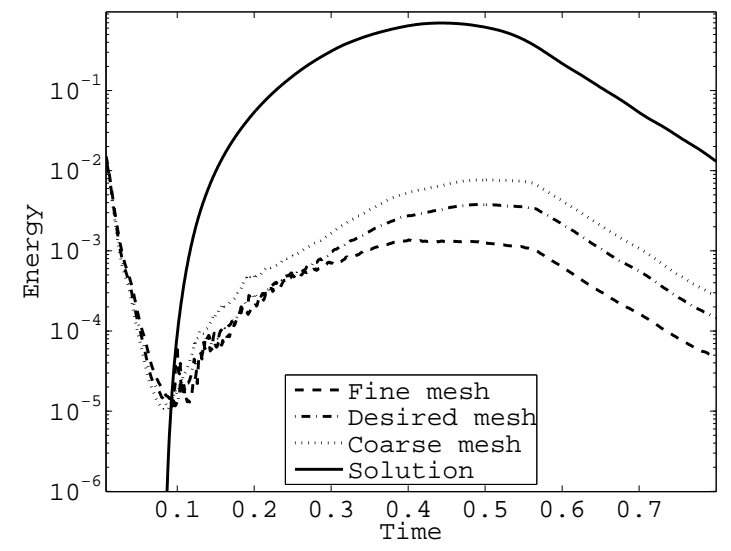

Figure 12: Global error with respect to the discretization (energy in $\log$ scale and $\gamma=2$ )

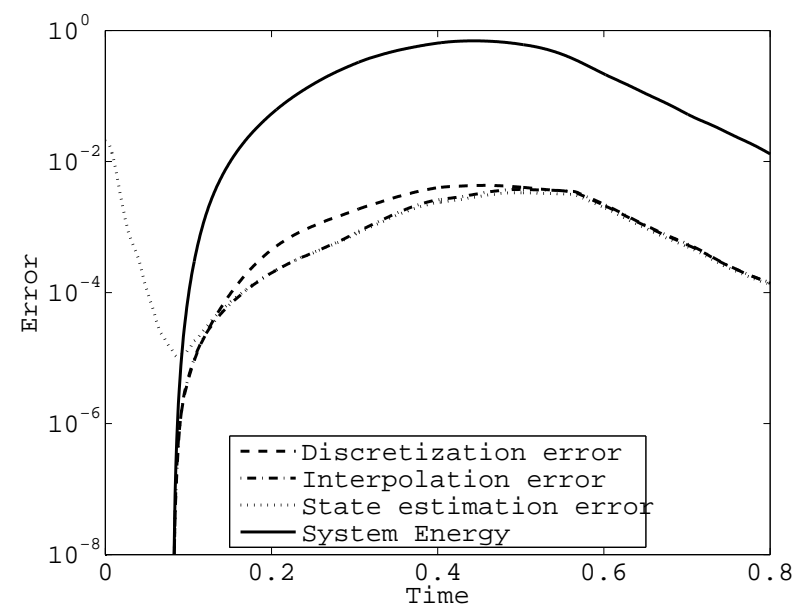

Figure 13: Comparison of estimation, interpolation and discretization errors

can write the following linear state-parameter system:

$$
\left\{\begin{array}{l}
\dot{x}=\mathcal{A} x+\mathcal{B} \theta+\mathcal{R} \\
x(0)=x_{0}+\zeta_{x} \\
\theta=\theta_{0}+\zeta_{\theta}
\end{array}\right.
$$

where $\zeta_{\theta}$ represents the unknown part in the parameters.

If the parameters were perfectly known we could apply the above state estimation procedure. Therefore, we have formally

$$
\forall \zeta_{\theta}, \quad \lim _{t \rightarrow \infty}\left\|\bar{x}_{h}\left(t, \zeta_{\theta}\right)-x\left(t, \zeta_{\theta}\right)\right\|=O\left(h^{p}\right)+\text { noise. }
$$

But since we do not know $\zeta_{\theta}$ the state-estimator $\bar{X}$ (and equivalently $\bar{x}_{h}$ ) is unusable as such. Our main objective here is to propose an estimator $\hat{X}$ which can start from $\theta_{0}-$ not $\theta_{0}+\zeta_{\theta}-$ and estimate the pair 
$\left(\zeta_{X}, \zeta_{\theta}\right)$. This is what we call joint state-parameter estimation, also referred to as adaptative observation in $[41,42]$.

\subsection{Construction and analysis of the estimation procedure}

Since we want to jointly estimate the state and the parameters, we could think of using filtering procedures - such as Kalman filtering - on the (discrete) system describing the evolution of the "augmented state" incorporating the parameters with the actual state (as described in [11]), namely,

$$
\left\{\begin{array}{l}
\dot{X}^{e}=A^{e} X^{e}+R^{e} \\
X^{e}(0)=X_{0}^{e}+\zeta^{e}
\end{array}\right.
$$

where

$$
X^{e}=\left(\begin{array}{c}
X \\
\theta
\end{array}\right), \quad A^{e}=\left(\begin{array}{cc}
A & B \\
0 & 0
\end{array}\right), \quad R^{e}=\left(\begin{array}{c}
R \\
0
\end{array}\right),
$$

and

$$
X_{0}^{e}=\left(\begin{array}{c}
X_{0} \\
\theta
\end{array}\right), \quad \zeta^{e}=\left(\begin{array}{c}
\zeta_{X} \\
\zeta_{\theta}
\end{array}\right) .
$$

However, the size of the augmented state vector $X^{e}$, namely, $2 n+r$, makes classical filtering techniques - which would also not take advantage of the state estimator introduced above - untractable for this problem. Thus, we aim at building a joint state-parameter estimator based on the "cheap" state estimator $\bar{X}$. Therefore we define the system describing the dynamics of $\bar{X}^{e}=\left(\begin{array}{ll}\bar{X} & \theta\end{array}\right)^{T}$, viz.

$$
\left\{\begin{array}{l}
\dot{\bar{X}}^{e}=A^{e} \bar{X}^{e}+R^{e}+K_{X}^{e}\left(Z-H^{e} \bar{X}^{e}\right) \\
\bar{X}^{e}(0)=X_{0}^{e}+\zeta_{\theta}^{e}
\end{array}\right.
$$

where

$$
\zeta_{\theta}^{e}=\left(\begin{array}{c}
0 \\
\zeta_{\theta}
\end{array}\right), \quad K_{X}^{e}=\left(\begin{array}{c}
K_{X} \\
0
\end{array}\right), \quad H^{e}=\left(\begin{array}{ll}
H & 0
\end{array}\right) .
$$

Here we point out that $\bar{X}^{e}$ only depends on the initial condition $\zeta_{\theta}$. Hence we can define $\bar{X}^{e}[\xi]$ for an arbitrary initial condition $\theta(0)=\theta_{0}+\xi$.

Our idea is to apply a Kalman filtering procedure on System (4.6). Two difficulties are to be noted in this respect: (1) $Z$ is not the observation corresponding to $\bar{X}^{e}$ but to $x$; (2) $Z$, which contains some (unknown) noise, is in the right-hand side of the system observed, hence this introduces some modeling noise in the state equation. In order to circumvent these difficulties in a first stage, we introduce the additional auxiliary system

$$
\left\{\begin{array}{l}
\dot{\bar{X}}_{a}^{e}=A^{e} \bar{X}_{a}^{e}+R^{e}+K_{X}^{e}\left(\bar{Z}-H^{e} \bar{X}_{a}^{e}\right) \\
\bar{X}_{a}^{e}(0)=X_{0}^{e}+\zeta_{\theta}^{e}
\end{array}\right.
$$

using the abstract observation without noise defined in (2.5). Considering also the "virtual" observation

$$
Z_{a}=H \bar{X}_{a}^{e}+\chi,
$$

we define $\hat{X}_{a}^{e}$ as the Kalman observer for this system (4.7) when using the virtual observation $Z_{a}$. The error covariance of this observer, namely,

$$
P_{a}^{e}=\mathbb{E}\left(\left(\bar{X}_{a}^{e}-\hat{X}_{a}^{e}\right)\left(\bar{X}_{a}^{e}-\hat{X}_{a}^{e}\right)^{T} \mid Z_{a}\right)
$$


is such that

$$
P_{a}^{e}(0)=\left(\begin{array}{cc}
0 & 0 \\
0 & \mathbb{E}\left(\zeta_{\theta} \zeta_{\theta}^{T}\right)
\end{array}\right) .
$$

Therefore, we are then in a position to apply Kalman filtering to a system with reduced rank covariance error. As shown in [32], in the absence of model noise, the covariance matrix at every time remains of constant rank $r$, namely, the size of the parameter vector. This leads to the so-called "Singular Evolutive Extended Kalman" (SEEK) algorithm in which the evolution equation of $P_{a}^{e}$ is:

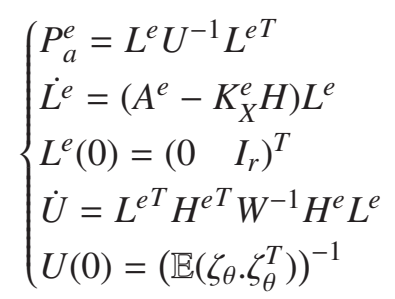

where $W$ denotes the covariance matrix of the observation noise $\chi$. If we decompose the equation describing $L^{e}$ as $L^{e}=\left(\begin{array}{ll}L_{X} & L_{\theta}\end{array}\right)^{T}$ we have in our case

$$
\left\{\begin{array}{l}
\dot{L}_{X}=\left(A-K_{X} H\right) L_{X}+B L_{\theta} \\
\dot{L}_{\theta}=0 \\
L_{X}(0)=0 \\
L_{\theta}(0)=I_{r}
\end{array}\right.
$$

Hence $L_{\theta}=I_{r}, \forall t$, which implies

$$
\dot{L}_{X}=\left(A-K_{X} H\right) L_{X}+B,
$$

and

$$
\dot{U}=L_{X}^{T} H^{T} W^{-1} H L_{X} .
$$

Finally, Kalman filtering specialized for the auxiliary system (4.7) gives the following observer equations

$$
\left\{\begin{array}{l}
\hat{\hat{X}}_{a}=A \hat{X}_{a}+B \hat{\theta}_{a}+R+K_{X}\left(\bar{Z}-H \hat{X}_{a}\right)+L_{X} \dot{\hat{\theta}}_{a} \\
\hat{\hat{\theta}}_{a}=U^{-1} L_{X}^{T} H^{T} W^{-1}\left(Z_{a}-H \hat{X}_{a}\right) \\
\hat{X}(0)=X_{0} \\
\hat{\theta}(0)=\theta_{0}
\end{array}\right.
$$

with $L_{X}$ and $U$ respectively given by (4.13) and (4.14), and we note that we only need to manipulate a matrix of size $r$ (namely, $U$ ), instead of a covariance matrix with the size of the extended state, which makes the computation of the filter tractable. We emphasize that this rank reduction was achieved by considering the (stabilized) state estimator instead of the original state equations in the augmented system (4.7), which in essence amounts to "canceling" the uncertainty associated with the state initial conditions. This differs from the SEEK approach per se, in which rank reduction is performed by retaining only the dominating singular values in the global covariance matrix. Here, the uncertainty considered has the same dimension as the complete parameter space.

Of course, the observer $\hat{X}_{a}$ cannot be used as such, since neither $\bar{Z}$ nor $Z_{a}$ are available. We then resort to the natural idea of substituting the actual measurements $Z$ for these two quantities, which provides the 
following system.

$$
\left\{\begin{array}{l}
\dot{\hat{X}}=A \hat{X}+B \hat{\theta}+R+K_{X}(Z-H \hat{X})+L_{X} \dot{\hat{\theta}} \\
\dot{\hat{\theta}}=U^{-1} L_{X}^{T} H^{T} W^{-1}(Z-H \hat{X}) \\
\dot{L}_{X}=\left(A-K_{X} H\right) L_{X}+B \\
\dot{U}=L_{X}^{T} H^{T} W^{-1} H L_{X} \\
\hat{X}(0)=X_{0} \\
\hat{\theta}(0)=\theta_{0} \\
L_{X}(0)=0 \\
U(0)=\left(\mathbb{E}\left(\zeta_{\theta} \cdot \zeta_{\theta}^{T}\right)\right)^{-1}
\end{array}\right.
$$

Note that $Z$ differs from $\bar{Z}$ only by the noise, and that $Z_{a}$ converges to a numerical approximation of $Z$ with the time constant of the collocated closed-loop system, see Section 2. Through this substitution we lose the "theoretical optimality" associated with Kalman filtering in the reduced covariance rank framework (namely, SEEK). However, we can still establish an optimality result in a variational context.

THEOREM 4.1

Considering the variational criterion

$$
J_{T}(\xi)=\frac{1}{2} \xi^{T} U(0) \xi+\frac{1}{2} \int_{0}^{T}\left(Z-H^{e} \bar{X}^{e}(\xi)\right)^{T} W^{-1}\left(Z-H^{e} \bar{X}^{e}(\xi)\right) d t,
$$

there exists a unique minimizer $\hat{\xi}$ and the system (4.16) is such that

$$
\hat{X}(T)=\bar{X}^{e}[\hat{\xi}](T), \quad \forall T \geq 0 .
$$

\section{$\diamond$ Proof :}

In order to obtain the minimizer, we differentiate $J_{T}$

$$
d J_{T}(\xi) \cdot h=\xi^{T} U(0) h-\int_{0}^{T}\left(Z-H^{e} \bar{X}^{e}\right)^{T} W^{-1} H^{e} \frac{d \bar{X}^{e}}{d \xi} \cdot h d t
$$

where

$$
\left\{\begin{array}{l}
\frac{d}{d t} \frac{d \bar{X}^{e}}{d \xi}=\left(A^{e}-K_{X}^{e} H^{e}\right) \frac{d \bar{X}^{e}}{d \xi} \\
\frac{d \bar{X}^{e}}{d \xi}(0)=\left(\begin{array}{ll}
0 & I_{r}
\end{array}\right)^{T}
\end{array}\right.
$$

Let us introduce the adjoint variable $p^{e}$ satisfying

$$
\left\{\begin{array}{l}
\dot{p}^{e}+\left(A^{e}-K_{X}^{e} H^{e}\right)^{T} p^{e}=H^{e T} W^{-1}\left(Z-H^{e} \bar{X}^{e}\right) \\
p^{e}(T)=0
\end{array}\right.
$$

Then,

$$
\begin{aligned}
d J_{T}(\xi) \cdot h= & \xi^{T} U(0) h-\int_{0}^{T}\left(\dot{p}^{e}+\left(A^{e}-K_{X}^{e} H^{e}\right)^{T} p^{e}\right)^{T} \frac{d \bar{X}^{e}}{d \xi} \cdot h d t \\
= & \xi^{T} U(0) h+\int_{0}^{T} p^{e T}\left(\frac{d}{d t} \frac{d \bar{X}^{e}}{d \xi}-\left(A^{e}-K_{X}^{e} H^{e}\right) \frac{d \bar{X}^{e}}{d \xi}\right) \cdot h d t \\
& -\left[p^{e T} \frac{d \bar{X}^{e}}{d \xi} \cdot h\right]_{0}^{T} \\
= & \left(\left(\begin{array}{ll}
0 & \left.\left.I_{r}\right) p^{e}(0)+U(0) \xi\right)^{T} \cdot h
\end{array}\right.\right.
\end{aligned}
$$


Defining $\xi^{e}=\left(\begin{array}{ll}0 & \xi\end{array}\right)^{T}$, so that $\bar{X}^{e}[\xi](0)=X_{0}^{e}+\xi^{e}$, we have for the minimizer

$$
\hat{\xi}^{e}=-P_{a}^{e}(0) p^{e}(0)
$$

and $\bar{X}_{\text {inf }}^{e}=\bar{X}^{e}[\hat{\xi}]$ verifies

$$
\left\{\begin{array}{l}
\dot{\bar{X}}_{\mathrm{inf}}^{e}=A^{e} \bar{X}_{\mathrm{inf}}^{e}+R^{e}+K_{X}\left(Z-H^{e} \bar{X}_{\mathrm{inf}}^{e}\right) \\
\dot{p}^{e}+\left(A^{e}-K_{X}^{e} H^{e}\right)^{T} p^{e}=H^{e T} W^{-1}\left(Z-H^{e} \bar{X}_{\mathrm{inf}}^{e}\right) \\
\bar{X}_{\mathrm{inf}}^{e}(0)=X_{0}^{e}-P_{a}^{e}(0) p^{e}(0) \\
p^{e}(T)=0
\end{array}\right.
$$

This is a two-point boundary value problem that we would like to express in a Cauchy problem form in order to obtain a recursive procedure. Let us seek a solution in a form such that an affine relation exists between $\bar{X}_{\text {inf }}^{e}$ and $p^{e}$ - in fact, such an affine relation can be proven to hold due to the linearity of (4.19), see [26]. Then there exists some $\left(r^{e}, P^{e}\right)$ such that

$$
\bar{X}_{\text {inf }}^{e}(t)=r^{e}(t)-P^{e}(t) p^{e}(t) .
$$

Substituting in (4.19) we obtain

$$
\begin{aligned}
& \dot{r}^{e}(t)+P^{e} H^{e T} W^{-1} H^{e} r^{e}-\left(A^{e}-K_{X}^{e} H^{e}\right) r^{e}-R^{e}-K_{X} Z \\
& \quad+\left(-\dot{P}^{e}+P^{e}\left(A^{e}-K_{X}^{e} H^{e}\right)^{T}+\left(A^{e}-K_{X}^{e} H^{e}\right) P^{e}-P^{e} H^{e T} W^{-1} H^{e} P^{e}\right) p^{e}=P^{e} H^{e} W^{-1} Z,
\end{aligned}
$$

Clearly, this equation and the condition at $t=0$ of (4.19) will be satisfied if $\left(r^{e}, P^{e}\right)$ are solutions of the system

$$
\left\{\begin{array}{l}
\dot{P^{e}}-P^{e}\left(A^{e}-K_{X}^{e} H^{e}\right)^{T}-\left(A^{e}-K_{X}^{e} H^{e}\right) P^{e}+P^{e} H^{e T} W^{-1} H^{e} P^{e}=0 \\
\dot{r}^{e}+P^{e} H^{e T} W^{-1} H^{e} r^{e}-\left(A^{e}-K_{X}^{e} H^{e}\right) r^{e}=P^{e} H^{e T} W^{-1} Z+R^{e}+K_{X} Z \\
P^{e}(0)=P_{a}^{e}(0) \\
r^{e}(0)=X_{0}^{e}
\end{array}\right.
$$

Then, for $\left(r^{e}, P^{e}\right)$ solutions of this system, substituting (4.20) in the second equation of (4.19) we can solve for $p^{e}$ and it is easy to check that the resulting $\bar{X}_{\text {inf }}^{e}$ solution obtained from (4.20) itself satisfies the first and third equations in (4.19) (respectively, the dynamics and initial condition). Therefore, with (4.21) and the affine relation (4.20) we obtain the solutions of the two-point boundary value problem (4.19).

We note that $r^{e}$ and $P^{e}$ are independent of the time interval $[0, T]$ and we can show by using the evolution equations of $L^{e}$ and $U^{e}$ and the initial condition of $P^{e}$ that

$$
\forall t \geq 0, \quad P^{e}=L^{e} U^{-1} L^{e T}=P_{a}^{e} .
$$

It is then straightforward to verify that $r^{e}$ satisfies the same Cauchy problem as $\hat{X}^{e}$, namely (4.16), hence

$$
\forall t \geq 0, \quad r^{e}=\hat{X}^{e},
$$

and at the end of a given minimization time interval,

$$
\hat{X}^{e}(T)=\bar{X}_{\text {inf }}^{e}(T) .
$$




\section{$\square$ REMARK :}

A direct interpretation of this result is that $\hat{\theta}$ represents the so-called "maximum a posteriori estimate" associated with the (parametrized) model given by the state estimator, see e.g. [29] for detailed discussions on this type of parameter estimation and other related procedures. Furthermore, although we have directly constructed our estimator using a spatially-discrete system, we point out that we would obtain a similar algorithm by considering the maximum a posteriori estimate for the DVF-stabilized continuous system and then discretizing the sensitivity dynamics (namely, $L_{X}$ in the discrete system) using the same finite element scheme as for the state equations.

\section{$\square$ REMARK :}

We note that our proposed estimation methodology - derived from (although not strictly equivalent to) the SEEK procedure - leads to observer equations that correspond to the adaptative observer method introduced in $[42,41]$. In other words, we have established - for a given choice of state estimator - an equivalence property between this adaptative observer strategy and the SEEK algorithm particularized to uncertainties restricted to parameters. An important advantage of our approach is that the variational framework provides a natural optimality criterion satisfied by the observer. Furthermore, the Kalman setting allows to obtain a time-discrete version of our procedure in a straightforward manner - in the classical prediction-correction form - with the corresponding time-discrete optimality result.

\subsection{Error analysis}

We now carry out the analysis of the errors defined by $\tilde{X}=X-\hat{X}, \tilde{\theta}=\theta-\hat{\theta}$. Following [42, 41], we introduce the auxiliary quantity

$$
\eta=\tilde{X}-L_{X} \tilde{\theta}
$$

Then

$$
\left\{\begin{array}{l}
\dot{\eta}=\left(A-K_{X} H\right) \eta-K_{X}\left(\epsilon_{h}+\chi\right) \\
\dot{\tilde{\theta}}=-U^{-1} L_{X}^{T} H^{T} W^{-1} H L_{X} \tilde{\theta}-U^{-1} L_{X}^{T} H^{T} W^{-1} H \eta-U^{-1} L_{X}^{T} H^{T} W^{-1}\left(\epsilon_{h}+\chi\right) \\
\eta(0)=\zeta_{X} \\
\tilde{\theta}(0)=\zeta_{\theta}
\end{array}\right.
$$

We note that $\eta$ follows exactly the same dissipative dynamics as the state-estimator error $\check{X}$, recall (3.1), hence the same conclusions as above hold. We now consider the dynamics of $\tilde{\theta}$, which we rewrite as

$$
\dot{\tilde{\theta}}=-U^{-1} L_{X}^{T} H^{T} W^{-1} H L_{X} \tilde{\theta}+U^{-1} L_{X}^{T} H^{T} \varrho,
$$

with

$$
\varrho=-W^{-1}\left(H \eta+\epsilon_{h}+\chi\right) .
$$

We have

$$
\frac{d}{d t}(U \tilde{\theta})=\dot{U} \tilde{\theta}+U\left(-U^{-1} L_{X}^{T} H^{T} W^{-1} H L_{X} \tilde{\theta}+U^{-1} L_{X}^{T} H^{T} \varrho\right)=L_{X}^{T} H^{T} \varrho
$$


taking into account the dynamics of $U$ given in (4.16). This gives

$$
\tilde{\theta}=U^{-1}\left(U(0) \tilde{\theta}(0)+\int_{0}^{t} L_{X}^{T} H^{T} \varrho d \tau\right) .
$$

Noting that

$$
U(t)=U(0)+\Upsilon(t),
$$

with

$$
\Upsilon(t)=\int_{0}^{t} L_{X}^{T} H^{T} W^{-1} H L_{X} d \tau
$$

we define $\lambda_{\text {inf }}(t)$ as the smallest solution of the generalized eigenvalue problem

$$
\Upsilon(t) \xi=\lambda U(0) \xi,
$$

and the norm in which we evaluate parameters (as in Eq.(4.17)) as

$$
\|\xi\|_{U(0)}=\left(\xi^{T} U(0) \xi\right)^{\frac{1}{2}} .
$$

Bounding $U(t)$ from below by $\left(1+\lambda_{\text {inf }}(t)\right) U(0)$ we infer from (4.26)

$$
\|\tilde{\theta}\|_{U(0)} \leq \frac{1}{1+\lambda_{\text {inf }}(t)}\left(\|\tilde{\theta}(0)\|_{U(0)}+\left\|U(0)^{-1} \int_{0}^{t} L_{X}^{T} H^{T} \varrho d \tau\right\|_{U(0)}\right) .
$$

The sensitivity matrix $L_{X}$ obeys the same dynamics as the damped state, hence it is stable. More specifically, we have a bound of the following type, for any set of parameters,

$$
\left\|L_{X}(t) \theta\right\|_{\mathcal{E}}^{2} \leq C \int_{0}^{t}\|B(\tau) \theta\|_{R H S}^{2} d \tau,
$$

where $\|\cdot\|_{R H S}$ denotes an adequate norm for the right-hand side of the mechanical system equation. Defining the following natural norms for $L_{X}$ and $B$

$$
\left\|L_{X}\right\|_{L}=\sup _{\|\theta\|_{U(0)}=1}\left\|L_{X} \theta\right\|_{\mathcal{E}}, \quad\|B\|_{B}=\sup _{\|\theta\|_{U(0)}=1}\|B \theta\|_{R H S},
$$

we thus obtain

$$
\left\|L_{X}(t)\right\|_{L}^{2} \leq C \int_{0}^{t}\|B(\tau)\|_{B}^{2} d \tau .
$$

We infer that the integral term in (4.28) is a sum of "small contributions" associated with the terms contained in $\varrho$ and controlled due to the above considerations. As a consequence, the convergence of the parameter estimation is mainly governed by the eigenvalue $\lambda_{\text {inf }}(t)$. In this respect, we note that $\Upsilon(t)$ is an observability grammian matrix for which $\lambda_{\text {inf }}(t)$ gives the best lower bound with respect to $U(0)$, namely,

$$
\Upsilon(t) \geq \lambda_{\text {inf }}(t) U(0)
$$

Therefore the growth of $\lambda_{\text {inf }}(t)$ - which is an increasing function of time - is related to observability considerations. 
We now analyze in more details the effect of the small contributions contained in $\varrho$ on the error bound (4.28). Starting with the deterministic term due to $\epsilon_{h}$, we have

$$
\left\|U(0)^{-1} \int_{0}^{t} L_{X}^{T} H^{T} W^{-1} \epsilon_{h} d \tau\right\|_{U(0)} \leq \int_{0}^{t}\left\|U(0)^{-1} L_{X}^{T} H^{T} W^{-1} \epsilon_{h}\right\|_{U(0)} d \tau,
$$

and

$$
\left\|U(0)^{-1} L_{X}^{T} H^{T} W^{-1} \epsilon_{h}\right\|_{U(0)}^{2}=\epsilon_{h}^{T} W^{-1} H L_{X} U(0)^{-1} L_{X}^{T} H^{T} W^{-1} \epsilon_{h}
$$

Noting that the induced norm defined on $L_{X}$ is related to Rayleigh quotients as follows

$$
\left\|L_{X}\right\|_{L}^{2}=\sup _{\theta \neq 0} \frac{\theta^{T} L_{X}^{T} N L_{X} \theta}{\theta^{T} U(0) \theta}=\sup _{X \neq 0} \frac{X^{T} N L_{X} U(0)^{-1} L_{X}^{T} N X}{X^{T} N X},
$$

we infer

$$
\begin{aligned}
\left\|U(0)^{-1} L_{X}^{T} H^{T} W^{-1} \epsilon_{h}\right\|_{U(0)}^{2} & \leq\left\|L_{X}\right\|_{L}^{2}\left(\epsilon_{h}^{T} W^{-1} H N^{-1} H^{T} W^{-1} \epsilon_{h}\right) \\
& \leq\left\|L_{X}\right\|_{L}^{2}\left(\epsilon_{h}^{T} W^{-1} \epsilon_{h}\right) \sup _{Y \neq 0} \frac{Y^{T} W^{-1} H N^{-1} H^{T} W^{-1} Y}{Y^{T} W^{-1} Y} \\
& =\left\|L_{X}\right\|_{L}^{2}\left\|\epsilon_{h}\right\|_{W^{-1}}^{2} \sup _{X \neq 0} \frac{X^{T} H^{T} W^{-1} H X}{X^{T} N X},
\end{aligned}
$$

using again an identity similar to (4.35). Invoking the continuity of the observation operator, viz.

$$
\|H X\|_{W^{-1}} \leq C_{\text {obs }}\|X\|_{\mathcal{E}}
$$

this leads to

$$
\left\|U(0)^{-1} \int_{0}^{t} L_{X}^{T} H^{T} W^{-1} \epsilon_{h} d \tau\right\|_{U(0)} \leq C_{\mathrm{obs}} \int_{0}^{t}\left\|L_{X}(\tau)\right\|_{L}\left\|\epsilon_{h}(\tau)\right\|_{W^{-1}} d \tau
$$

Note that we also have, recalling (2.12),

$$
\left\|\epsilon_{h}(\tau)\right\|_{W^{-1}} \leq C_{\mathrm{obs}}\left\|x-x_{h}\right\|_{\mathcal{E}}
$$

using the same notation for the energy norm applied in the discrete and continuous state spaces. Therefore,

$$
\left\|U(0)^{-1} \int_{0}^{t} L_{X}^{T} H^{T} W^{-1} \epsilon_{h} d \tau\right\|_{U(0)} \leq\left(C_{\mathrm{obs}}\right)^{2} \int_{0}^{t}\left\|L_{X}(\tau)\right\|_{L}\left\|\left(x-x_{h}\right)(\tau)\right\|_{\mathcal{E}} d \tau .
$$

Similarly, for the contribution of $\eta$ in $\varrho$ we obtain

$$
\begin{aligned}
\left\|U(0)^{-1} \int_{0}^{t} L_{X}^{T} H^{T} W^{-1} H \eta d \tau\right\|_{U(0)} & \leq C_{\mathrm{obs}} \int_{0}^{t}\left\|L_{X}(\tau)\right\|_{L}\|H \eta(\tau)\|_{W^{-1}} d \tau \\
& \leq\left(C_{\mathrm{obs}}\right)^{2} \int_{0}^{t}\left\|L_{X}(\tau)\right\|_{L}\|\eta(\tau)\|_{\mathcal{E}} d \tau .
\end{aligned}
$$

Note that this bound allows to handle both the deterministic and probabilistic parts of $\eta$. In particular, we can obtain the bound for the term due to the probabilistic part in $\eta$ by using the estimate (3.9), also applicable for $\eta$. 
Finally, considering the random part in the right-hand side of the error bound (4.28) due to the white noise in $\varrho$, we have zero mean - of course - and the covariance of the norm gives:

$$
\begin{aligned}
\mathbb{E}\left(\left\|U(0)^{-1} \int_{0}^{t} L_{X}^{T} H^{T} W^{-1} \chi d \tau\right\|_{U(0)}^{2}\right) & =\mathbb{E}\left(\int_{0}^{t} \chi^{T} W^{-1} H L_{X} U(0)^{-1} L_{X}^{T} H^{T} W^{-1} \chi d \tau\right) \\
& =\mathbb{E}\left(\int_{0}^{t} \operatorname{Tr}\left(U(0)^{-1} L_{X}^{T} H^{T} W^{-1} \chi \chi^{T} W^{-1} H L_{X}\right) d \tau\right) \\
& =\int_{0}^{t} \operatorname{Tr}\left(U(0)^{-1} L_{X}^{T} H^{T} W^{-1} Q_{\chi} W^{-1} H L_{X}\right) d \tau \\
& =\sum_{i=1}^{q}\left(\alpha_{i}\right)^{2} \sum_{j=1}^{3} \int_{0}^{t}\left(V_{i}^{j}\right)^{T} W^{-1} H L_{X} U(0)^{-1} L_{X}^{T} H^{T} W^{-1} V_{i}^{j} d \tau,
\end{aligned}
$$

where we used the decomposition (2.6) and standard trace properties. Then, using similar arguments as for the deterministic contributions we obtain

$$
\mathbb{E}\left(\left\|U(0)^{-1} \int_{0}^{t} L_{X}^{T} H^{T} W^{-1} \chi d \tau\right\|_{U(0)}^{2}\right) \leq C_{\mathrm{obs}}^{2}\left(\sum_{i=1}^{q}\left(\alpha_{i}\right)^{2} \sum_{j=1}^{3}\left\|V_{i}^{j}\right\|_{W^{-1}}^{2}\right) \int_{0}^{t}\left\|L_{X}(\tau)\right\|_{L}^{2} d \tau .
$$

We can now summarize the convergence of parametric estimation as follows, combining the above estimates and (3.13).

$$
\begin{aligned}
\mathbb{E}\left(\|\tilde{\theta}\|_{U(0)}^{2}\right) \leq \frac{C}{\left(1+\lambda_{\mathrm{inf}}(t)\right)^{2}}\left\{1+\left\|L_{X}\right\|_{L^{2}([0, T] ; L)}^{2}\left[C_{\mathrm{obs}}^{2}\left(\sum_{i=1}^{q}\left(\alpha_{i}\right)^{2} \sum_{j=1}^{3}\left\|V_{i}^{j}\right\|_{W^{-1}}^{2}\right)\right.\right. \\
\left.\left.+\left(C_{\mathrm{obs}}\right)^{4}\left(T_{1} \mathbb{E}\left(\left\|\zeta_{X}\right\|_{\mathcal{E}}^{2}\right)+\left(1+t T_{2} \gamma^{2}\right)\left\|x-x_{h}\right\|_{L^{2}([0, T] ; \mathcal{E})}^{2}+t T_{2} \gamma^{2} \sum_{i=1}^{q} \alpha_{i}^{2}+t \varepsilon^{2}\right)\right]\right\} \cdot
\end{aligned}
$$

With the above error estimate for the parametric estimation combined with the bound (3.13) which also holds with $\eta$ substituted for $\check{X}$, it is now straightforward to derive an error estimate for the state estimation, recalling that $\eta=\tilde{X}-L_{X} \tilde{\theta}$. Namely, we simply have

$$
\|\tilde{X}\|_{\mathcal{E}} \leq\|\eta\|_{\mathcal{E}}+\left\|L_{X}\right\|_{L}\|\tilde{\theta}\|_{U(0)}
$$

\section{$\square$ REMARK :}

As already pointed out, the smallest eigenvalue of the matrix $\Upsilon(t)$ is related to observability. In fact, this matrix can be interpreted as a parameter-related observability Grammian matrix, as is classically considered in control theory, see e.g. [30]. Furthermore, it can be noted that the above error analysis still holds when changing the observation operator used in the parameter observer dynamics, namely, in the 2nd line of System (4.16). Hence, the observation operator $H$ appearing in the observability Grammian can be different from that used in the state estimator, and in particular incorporate additional measurements in order to improve identifiability.

We show in Figures 14-19 the numerical results obtained with our joint estimation procedure for the test problem of Section 2 when seeking to identify the contractility parameters $\sigma_{0}$ in the AHA regions. 
In these computations the initial covariance for the contractility parameters was set as $\mathbb{1}_{17 \times 17}$ - which corresponds to the actual uncertainty that we aim at representing - and the state observer gain as $\gamma=2$. We can see that the values of the contractility parameters are quite accurately and rapidly estimated, even though the accuracy significantly depends on the modeling error. On the other hand, state estimation is much more accurate - and also much faster - and indeed is not very different from the behaviour observed in the previous section (namely, state estimation by itself).

\section{Joint state and parameter bilinear estimation}

In the previous sections we have dealt with the estimation of prestress parameters, and this problem led to a linear dynamical system in the combined state and parameter variables. However, this linearity only holds when the parameters appear in the "right-hand side" of the dynamical system. When considering the estimation of other mechanical parameters - and typically that of constitutive parameters - the resulting augmented system is in general non-linear, even if the mechanical system by itself obeys a linear dynamics.

In this framework, let us consider that we want to determine unknown variations of Young's modulus with respect to a reference value. Keeping our above subdivision of $\Omega$ in 17 regions where the parameters are assumed to be constant, we can write the following bilinear augmented state-parameter system

$$
\left\{\begin{array}{l}
\dot{x}=(\mathcal{A}+\Delta \mathcal{A} . \theta) x+\mathcal{R} \\
x(0)=x_{0}+\zeta_{x} \\
\theta=\theta_{0}+\zeta_{\theta}
\end{array}\right.
$$

where we can decompose $\Delta \mathcal{A}$ using the canonical basis for the parameter space $a_{i}^{\theta}=\left(\delta_{i j}\right)_{1 \leq j \leq 17}$, namely,

$$
\Delta \mathcal{A}=\sum_{i=1}^{17} \Delta \mathcal{A}_{i} \otimes a_{i}^{\theta} \Longleftrightarrow \Delta \mathcal{A} . \theta=\sum_{i=1}^{17} \Delta \mathcal{A}_{i} \theta_{i}
$$

We can also explicitly write the corresponding second-order discrete dynamical equation as

$$
M \ddot{Y}+C \dot{Y}+(K+\Delta K \cdot \theta) Y=F,
$$

where $F$ may contain the prestress contribution as above.

Since the mechanical system by itself is linear, the above-described state estimator is applicable, and we can directly focus on extending our results on parameter estimation in the augmented system. Although Kalman filtering is optimal for linear systems only, extended algorithms based on linearized operators may lead to efficient - albeit non-optimal - filtering procedures [3]. Here, we present an original interpretation of our previous algorithm in order to find an extended version. To this end, we reconsider the equations on the state estimator $\bar{X}$ - recall (2.17) - and on the quantity $L_{X}$ defined in the linear framework in (4.16), namely,

$$
\left\{\begin{array}{l}
\dot{\bar{X}}=A \bar{X}+K_{X}(Z-H \bar{X})+B \theta+R \\
X(0)=X_{0}+\zeta_{X} \\
\dot{L}_{X}=\left(A-K_{X} H\right) L_{X}+B \\
L_{X}(0)=0
\end{array}\right.
$$




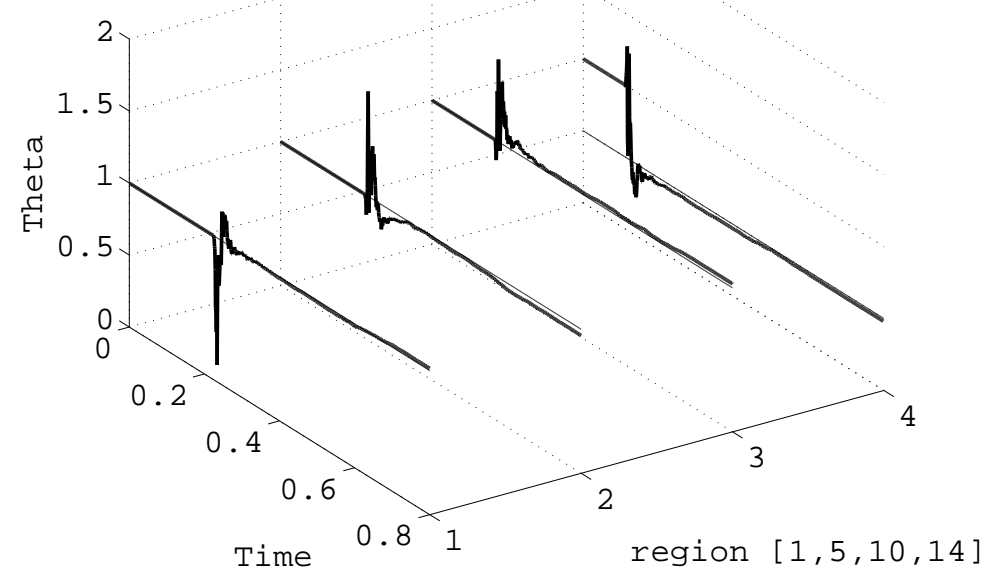

Figure 14: Convergence of 4 contractility parameters corresponding to regions $[1,5,10,14]$.

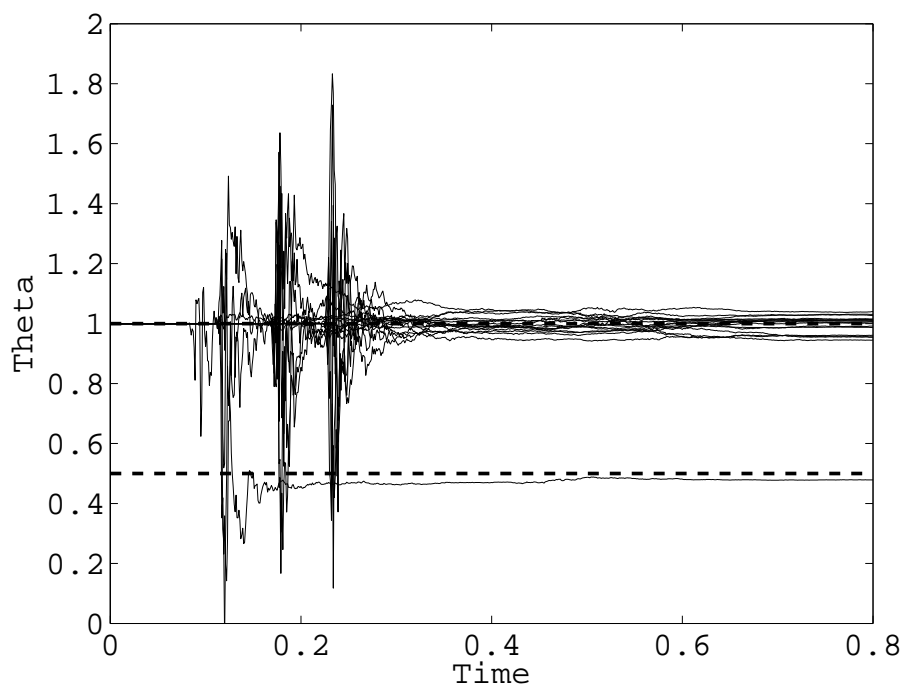

Figure 15: Estimated values of the 17 contractility parameters. 


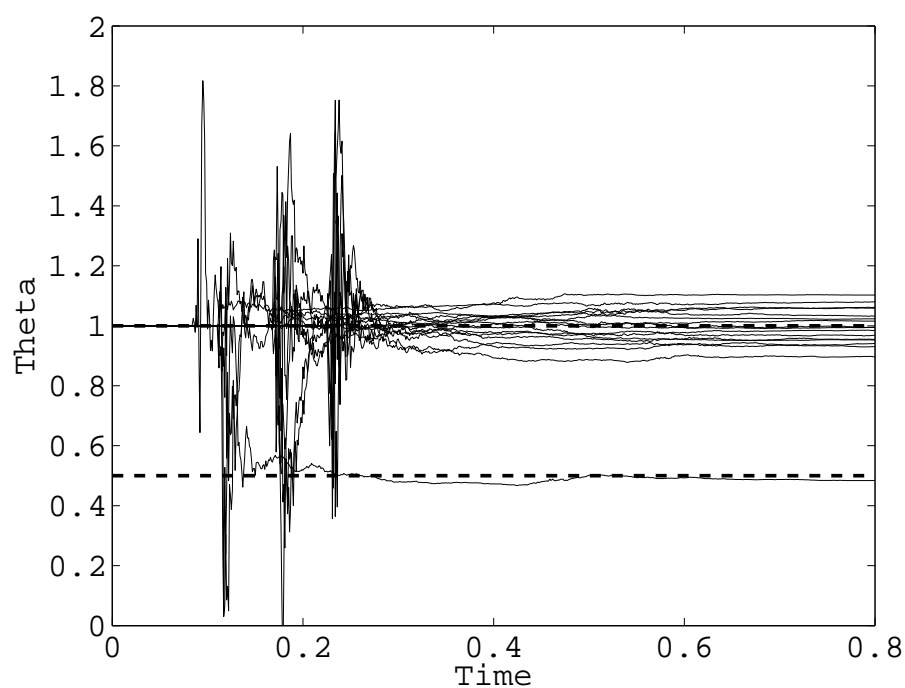

Figure 16: Estimated values of the 17 contractility parameters with the coarse mesh.

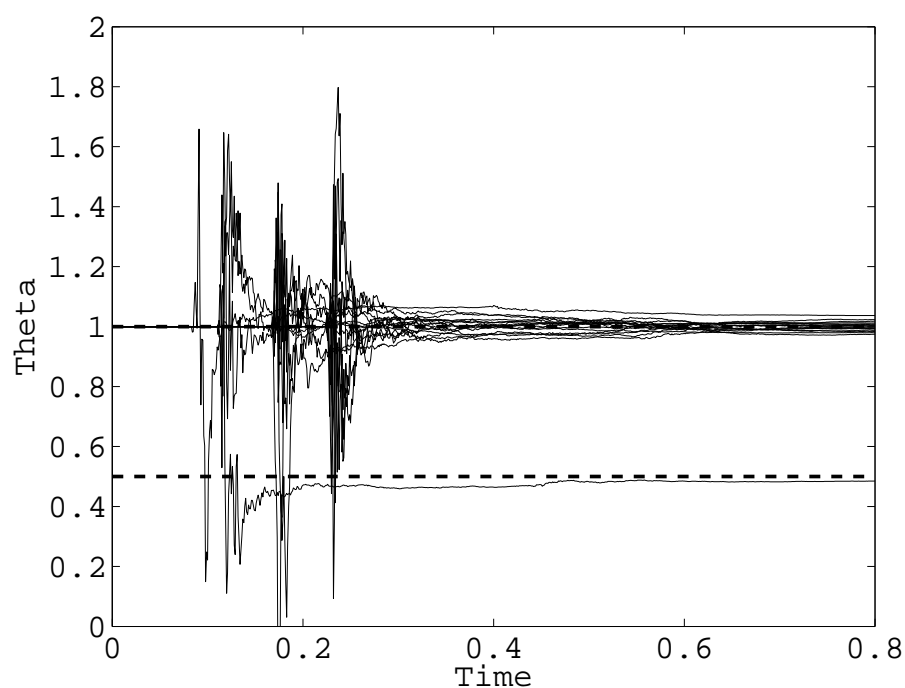

Figure 17: Estimated values of the 17 contractility parameters with the fine mesh. 


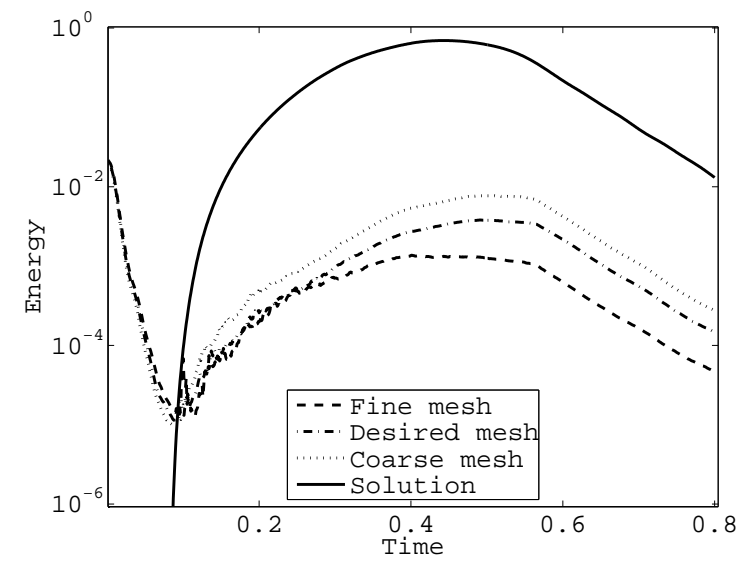

Figure 18: State error in joint estimation with respect to the discretization.
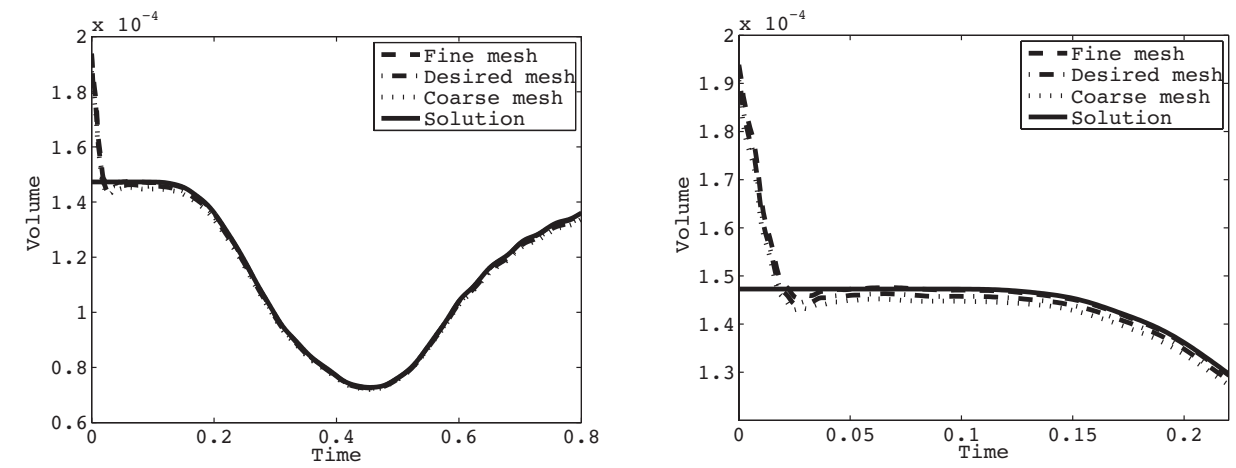

Figure 19: Estimated volume with respect to the discretization. 
We note that $L_{X}$ can be interpreted as the sensitivity of the state estimator $\bar{X}$ with respect to $\theta$, i.e. $L_{X}=\frac{d \bar{X}}{d \theta}$. From this interpretation we can devise an observer in the bilinear case by differentiating the first line of (5.3), which gives

$$
\left\{\begin{array}{l}
\dot{\hat{X}}=A \hat{X}+\Delta A \cdot \hat{\theta} \hat{X}+R+K_{X}(Z-H \hat{X})+L_{X} \dot{\hat{\theta}} \\
\dot{\hat{\theta}}=U^{-1} L_{X}^{T} H^{T} W^{-1}(Z-H \hat{X}) \\
\dot{L}_{X}=\left(A+\Delta A \cdot \hat{\theta}-K_{X} H\right) L_{X}+\Lambda \hat{X} \\
\dot{U}=L_{X}^{T} H^{T} W^{-1} H L_{X} \\
\hat{X}(0)=X_{0} \\
\hat{\theta}(0)=\theta_{0} \\
L_{X}(0)=0 \\
U(0)=\left(\mathbb{E}\left(\zeta_{\theta} \cdot \zeta_{\theta}^{T}\right)\right)^{-1}
\end{array}\right.
$$

where

$$
\Lambda X=\partial_{\theta}([\Delta A X] \theta)=\sum_{i} \Delta A_{i} X \otimes a_{i}^{\theta} .
$$

Compared to the system (4.16) describing the dynamics of the observer in the linear case, we have included the additional term $\Delta A . \hat{\theta}$ in the equation governing $L_{X}$ to take into account the dependence of the stiffness matrix with respect to the unknown parameters $\theta$.

$\square$ REMARK :

This estimation scheme can also be obtained by formally applying extended Kalman filtering (EKF, see [19]) on the augmented bilinear system with the "virtual" measurement $Z_{a}$, similarly to the Kalman filtering construction that we presented for the linear case in Section 4.2, namely, by using the linearized dynamics in the construction of the filter.

Let us now analyze the convergence of this algorithm. We still rewrite the system satisfied by the error $(\tilde{X}, \tilde{\theta})$ using the change of variables into $(\eta, \tilde{\theta})$. A straightforward computation leads to

$$
\left\{\begin{array}{l}
\dot{\eta}=\left(A+\Delta A \theta-K_{X} H\right) \eta+\Delta A \tilde{\theta} L_{X} \tilde{\theta}+K_{X}\left(\epsilon_{h}+\chi\right) \\
\dot{\tilde{\theta}}=-U^{-1} L_{X}^{T} H^{T} W^{-1} H L_{X} \tilde{\theta}-U^{-1} L_{X}^{T} H^{T} W^{-1} H \eta-U^{-1} L_{X}^{T} H^{T} W^{-1}\left(\epsilon_{h}+\chi\right) \\
\eta(0)=\zeta_{X} \\
\tilde{\theta}(0)=\zeta_{\theta}
\end{array}\right.
$$

which has lost its linearity. But the associated tangent system in $(\eta, \tilde{\theta})=(0,0)$ remains exactly as System (4.25) of which we have proven the stability in the previous section. From the classical theory of stability of non linear systems [35, 34], we infer that there exists a neighbourhood of $(\eta, \tilde{\theta})=(0,0)-$ hence of $(\tilde{X}, \tilde{\theta})=(0,0)-$ such that System 5.5 remains stable. As a consequence, we expect to accurately achieve our joint estimation if the indeterminations $\zeta_{X}$ et $\zeta_{\theta}$ are "small".

\section{$\square$ REMARK :}

The collocated state estimator belongs to the class of "deterministic models" as defined in [28], hence the asymptotic (in time) convergence results obtained in this reference for extended Kalman filtering applied to such models hold in our case, namely, the parameter estimates converge to values which maximize a likelihood functional, provided they are kept (using a suitable projection algorithm) in a set for which the collocated system remains stable. 
We have tested our observer in a configuration quite similar to the one described in the linear case, and we used the same measurement operator. In the 14th AHA region we set $\theta_{14}=0.3$, while we let $\theta=0$ in the other regions, which means that the infarct produces an increase of the stiffness in the region concerned. Keeping the same initial error produced by a pressure of $1000 \mathrm{~Pa}$ on the endocardium, however, we did not directly obtain convergence. In our computations, indeed, we only achieved convergence with an initial error corresponding to a pressure 100 times lower, which reflects the above local convergence property, but is not satisfactory for practical purposes. Nevertheless, we know from the spectral analysis of Section 2.4 that the state observer $\bar{X}$ can estimate the state with an accuracy comparable to this reduced initial error in a very short time (about $0.1 \mathrm{~s}$ in our case). Furthermore, this convergence is robust with respect to the parameters, which means that for a quite large range of $\theta=\xi, \bar{X}(\xi)$ the observer can reach the desired neighbourhood in a comparable period of time.

Therefore, we propose to begin the estimation procedure with state estimation only, and to start the actual joint estimation after a delay $t_{s}$ calibrated based on the spectral analysis of the state estimator, recall Section 2.4. We remark that this proposed modified procedure can be simply written - and implemented in a straightforward manner - as

$$
\left\{\begin{array}{l}
\dot{\hat{X}}=A \hat{X}+\Delta A \cdot \hat{\theta} \hat{X}+R+K_{X}(Z-H \hat{X})+L_{X} \dot{\hat{\theta}} \\
\dot{\hat{\theta}}=U^{-1} L_{X}^{T} H^{T} W^{-1}(Z-H \hat{X}) \\
\dot{L}_{X}=0, \quad \text { if } t \leq t_{s} \\
\dot{L}_{X}=\left(A+\Delta A \cdot \hat{\theta}-K_{X} H\right) L_{X}+\Lambda \hat{X}, \quad \text { if } t>t_{s} \\
\dot{U}=L_{X}^{T} H^{T} W^{-1} H L_{X} \\
\hat{X}(0)=X_{0} \\
\hat{\theta}(0)=\theta_{0} \\
L_{X}(0)=0 \\
U(0)=\left(\mathbb{E}\left(\zeta_{\theta} \cdot \zeta_{\theta}^{T}\right)\right)^{-1}
\end{array}\right.
$$

In terms of computational complexity, compared to the state equation itself - and also to the observer equation in the linear case - the governing matrix in this new observer equation - namely, $A+\Delta A . \hat{\theta}-K_{X} H$ - is not constant in time, hence leads to a different matrix factorization for each time step. Nevertheless, the matrix to be factorized can be pre-assembled by parts for effective assembling at each step, and the resulting factors are then used for the equations that govern both $\hat{X}$ and the sensitivity $L_{X}$.

We display the corresponding numerical results in Fig. 20 and 21. The delay parameter was set as $t_{s}=0.1 \mathrm{~s}$, and the stiffness parameter covariance was taken as 0.04 in the regions numbered $7-10$ and 13-17 - i.e. Region 14 and the neighbouring parts - and very small in all other regions to represent an approximate "a priori prediction" of the infarcted area, and to enhance identifiability.

\section{$\square$ REMARK :}

The fact that parameter convergence is not obtained in this case without using the above delay in the parameter estimation startup confirms the crucial need for an effective and robust state estimation procedure to carry out joint state-parameter estimation. This can be interpreted by recalling our discussion on the optimization criterion associated with this estimation strategy, namely, that we minimize the error between the measurements and the state estimator $\bar{X}$, which can be successful only if the state estimator is sufficiently close to the real state. This is a general difficulty for parameter estimation problems, but in the special case of linear estimation the state $X$ did not enter in the dynamics of the parameter sensitivity $L_{X}$, hence an error on the state did not induce an error on the sensitivity, which made the joint estimation 


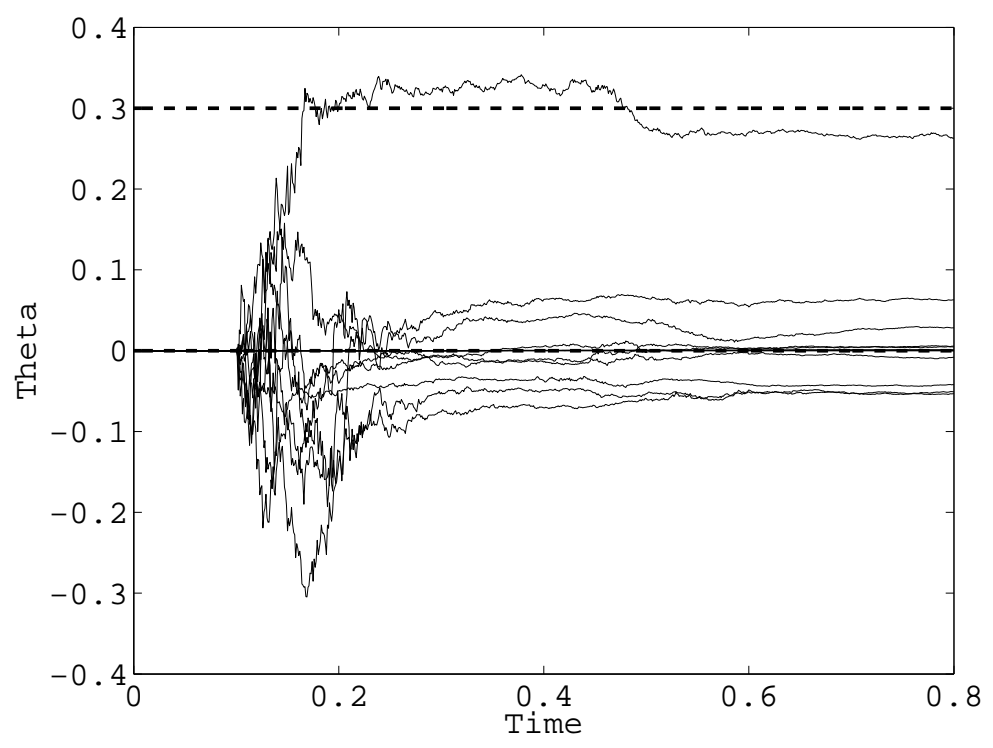

Figure 20: Estimated values of the 17 stiffness parameters

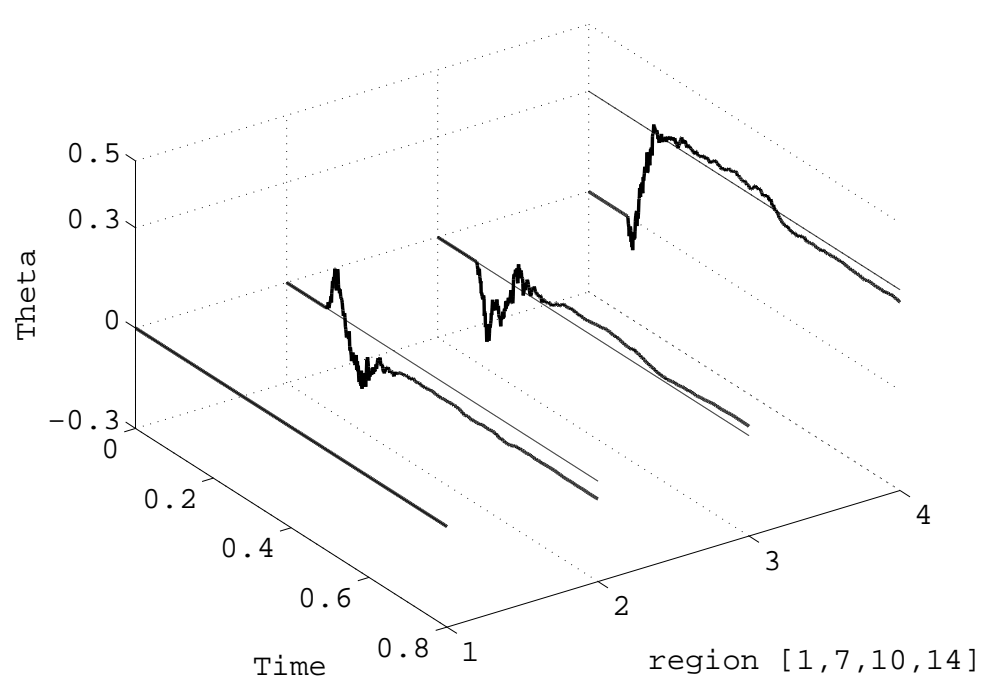

Figure 21: Convergence of 4 stiffness parameters corresponding to regions $[1,7,10,14]$. 
simpler and more effective.

\section{Concluding remarks}

We have proposed a joint state-parameter estimation procedure specifically adapted to the class of physical models of interest - which includes dynamically active soft tissues in biomechanics, in particular - and to the fundamental features of available measurement techniques, with an emphasis on imaging modalities. This estimator was built based on an effective and robust state estimation sequential strategy, namely, collocated feedback, which provides an estimation at the same computational cost as the simulation of the system itself.

Thus, in essence our estimation strategy consists in using physics-based estimators for the state variables, and rely on Kalman-like filtering only for the remaining variables in the augmented system, namely, the parameters for which the dynamics is non-physical.

We have demonstrated the performance of this procedure to estimate parameters of primary interest, namely, activation and stiffness parameters. For the stiffness estimation problem we had to consider a non-linear dynamical system, which required an extension of the methodology and an adaptation of the parameter estimation dynamics with the introduction of a startup delay. This necessary adaptation confirms that parameter estimation cannot be considered independently of state estimation, hence that a robust and effective state estimation procedure is an essential prerequisite.

Although the performance of the joint state-parameter estimator crucially depends on the effectiveness of the state filter considered, we emphasize that the methodology proposed to extend state estimation to joint state-parameter estimation is general and - indeed - applicable with any state filter. Other variants could also be constructed by considering for parameter estimation other types of nonlinear filtering than EKF- such as unscented Kalman filtering [20, 40] - or recursive identification algorithms, see e.g.[29].

Of course, biomechanical models frequently involve large displacements - as for cardiac mechanics - hence further work is required to extend our proposed methodology to nonlinear state equations. We also point out that other nonlinearities may arise from the observation operator, e.g. for the segmentation of contours in imaging measurements.

Furthermore, boundary measurements require a different approach to model measurement errors, because a white noise applied on the boundary of a mechanical system can be shown to induce solutions for which the mean energy is unbounded, in general. Indeed, in the case of boundary measurements - e.g., for ultrasound scans post-processed by optical flow techniques, see [31] - it is then more natural to set the estimation problem in the $H_{\infty}$ formalism (see [16]) where the space of measurement errors can be adequately defined.

We finally point out that further work is under way to evaluate the effectiveness of the proposed methodology when using synthetic measurements - namely, measurements produced by the model considered, as in this paper - specifically generated with physical models of real imaging modalities available in cardiology [14]. Using synthetic measurements allows to circumvent modeling difficulties and concentrate on the estimation per se. Nevertheless, once it has been validated with synthetic measurements accurately representing real data, the estimation procedure can also be used as a tool to assess model validity, since its success would then only depend on model adequacy.

Acknowledgment: The authors are thankful to Frédéric Bourquin (Laboratoire Central des Ponts et Chaussées) for several helpful discussions on collocated stabilization. 


\section{References}

[1] AHA/ACC/SNM. Standardization of cardiac tomographic imaging. Circulation, 86:338-339, 1992.

[2] Fatiha Alabau and Vilmos Komornik. Boundary observability, controllability, and stabilization of linear elastodynamic systems. SIAM J. Control Optim., 37(2):521-542, 1999.

[3] B. D. O. Anderson and J. B. Moore. Optimal Filtering. Prentice Hall, 1979.

[4] K.F. Augenstein, B.R. Cowan, I.J. LeGrice, and A.A. Young. Estimation of cardiac hyperelastic material properties from MRI tissue tagging and diffusion tensor imaging. In MICCAI, pages 628635, 2006.

[5] H. T. Banks and R. H. Fabiano. Approximation issues for applications in optimal control and parameter estimation. In Modelling and computation for applications in mathematics, science, and engineering (Evanston, IL, 1996), Numer. Math. Sci. Comput., pages 141-165. Oxford Univ. Press, New York, 1998.

[6] C. Bardos, G. Lebeau, and J. Rauch. Sharp sufficient conditions for the observation, control, and stabilization of waves from the boundary. SIAM J. Control Optim., 30(5):1024-1065, 1992.

[7] K.J. Bathe. Finite Element Procedures. Prentice Hall, 1996.

[8] A. Bensoussan. Filtrage optimal des systèmes linéaires. Dunod, 1971.

[9] H.D. Bui. Inverse Problems in the Mechanics of Materials: An Introduction. CRC Press, 1994.

[10] M. Collet, V. Walter, and P. Delobelle. Active damping of a micro-cantilever piezo-composite beam. J. Sound Vibration, 260(3):453-476, 2003.

[11] A. Corigliano and S. Mariani. Parameter identification in explicit structural dynamics: performance of the extended Kalman filter. Computer Methods in Applied Mechanics and Engineering, 193:3807-3835, 2004.

[12] S. Cox and E. Zuazua. The rate at which energy decays in a damped string. Comm. Partial Differential Equations, 19(1-2):213-243, 1994.

[13] R.F. Curtain and H. Zwart. An introduction to infinite-dimensional linear systems theory, volume 21 of Texts in Applied Mathematics. Springer-Verlag, New York, 1995.

[14] Q. Duan, Ph. Moireau, E. Angelini, D. Chapelle, and A.F. Laine. Simulation of 3D ultrasound with a realistic electro-mechanical model of the heart. In Proceedings of FIMH'07 Conference. Springer Verlag, 2007.

[15] Y.C.. Fung. Biomechanics: Mechanical properties of Living Tissues, second edition. SpringerVerlag, 1993.

[16] B. Hassibi, A.H. Sayed, and T. Kailath. Indefinite-quadratic estimation and control, volume 16 of SIAM Studies in Applied Mathematics. Society for Industrial and Applied Mathematics (SIAM), 1999. A unified approach to $H^{2}$ and $H^{\infty}$ theories. 
[17] G.A. Holzapfel and R.W. Ogden, editors. Mechanics of Biological Tissue. Springer Verlag, 2006.

[18] T.J.R. Hughes. The Finite Element Method : Linear Static and Dynamic Finite Element Analysis. Prentice Hall, 1987.

[19] A.H. Jazwinsky. Stochastic Processes and Filtering Theory. Academic Press, 1970.

[20] S. Julier and J. Uhlmann. Unscented filtering and nonlinear estimation. In Proceedings of the IEEE, volume 92, 2004.

[21] R.E. Kalman and R.S. Bucy. New results in linear filtering and prediction theory. ASME Trans.Journal of Basic Engineering, 83(Series D):95-108, 1961.

[22] E. Laporte and P. Le Tallec. Numerical Methods in Shape Optimisation and in Sensitivity Analysis. Birkhauser, 2002.

[23] F.X. Le Dimet and O. Talagrand. Variational algorithms for analysis and assimilation of meteorological observation : theoretical aspects. Tellus, 38:97-110, 1986.

[24] G. Lebeau. Équation des ondes amorties. In Algebraic and geometric methods in mathematical physics (Kaciveli, 1993), volume 19 of Math. Phys. Stud., pages 73-109. Kluwer Acad. Publ., Dordrecht, 1996.

[25] G. Lebeau and E. Zuazua. Decay rates for the three-dimensional linear system of thermoelasticity. Arch. Ration. Mech. Anal., 148(3):179-231, 1999.

[26] J.-L. Lions. Contrôle optimal de systèmes gouvernés par des équations aux dérivées partielles. Dunod, Paris, 1968.

[27] J.-L. Lions. Contrôlabilité exacte, perturbations et stabilisation de systèmes distribués. (Tome 1), volume 8 of Recherches en Mathématiques Appliquées [Research in Applied Mathematics]. Masson, Paris, 1988.

[28] L. Ljung. Asymptotic behavior of the extended Kalman filter as parameter estimator for linear systems. IEEE Transactions on Automatic Control, AC-24(1):36-50, 1979.

[29] L. Ljung. Theory and Practice of Recursive Identification. MIT Press, 1983.

[30] D.G Luenberger. An introduction to observers. IEEE Transactions on Automatic Control, 16:596602, 1971.

[31] X. Papademetris, A. J. Sinusas, D. P. Dione, and J. S. Duncan. Estimation of 3D left ventricular deformation from echocardiography. Medical Image Analysis, 8:285-294, 2004.

[32] D.T Pham, J. Verron, and M.C. Roubeaud. A singular evolutive interpolated Kalman filter for data assimilation in oceanography. J. Marine Systems, 16:323-341, 1997.

[33] A. Preumont. Vibration Control of Active Structures, An Introduction. Kluwer Academic Publishers, 2nd edition, February 2002.

[34] N. Rouche and J. Mawhin. Ordinary differential equations, volume 5 of Surveys and Reference Works in Mathematics. Pitman (Advanced Publishing Program), 1980. 
[35] Nicolas Rouche, P. Habets, and M. Laloy. Stability theory by Liapunov's direct method. Applied Mathematical Sciences, Vol. 22. Springer-Verlag, New York, 1977.

[36] J. Sainte-Marie, D. Chapelle, R. Cimrman, and M. Sorine. Modeling and estimation of the cardiac electromechanical activity. Comp. E Struct., 84:1743-1759, 2006.

[37] M. A. Shubov. The Riesz basis property of the system of root vectors for the equation of a nonhomogeneous damped string: transformation operators method. Methods Appl. Anal., 6(4):571-591, 1999.

[38] N.P. Smith, D.P. Nickerson, E.J. Crampin, and P.J. Hunter. Computational mechanics of the heart. from tissue structure to ventricular function. J. of Elasticity, 61(1):113-141, 2000.

[39] S. Tong and P. Shi. Cardiac motion recovery: Continuous dynamics, discrete measurements, and optimal estimation. In MICCAI, pages 744-751, 2006.

[40] M. Wu and A.W. Smyth. Application of the unscented Kalman filter for real-time nonlinear structural system identification. Struct. Control Health Monit. (in press).

[41] Q. Zhang. Adaptive observer for mimo linear time varying systems,. IEEE Transactions on Automatic Control, 3:525-529, March 2002.

[42] Q. Zhang and A. Clavel. Adaptive observer with exponential forgetting factor for linear time varying systems. In Decision and Control, 2001. Proceedings of the 40th IEEE Conference on, volume 4, pages 3886-3891, Orlando, FL, USA, 2001. 